\title{
A Modified Electrochemical Model to Account for Mechanical Effects Due to Lithium Intercalation and External Pressure
}

To cite this article before publication: Xiaoxuan Zhang et al $2021 \mathrm{~J}$. Electrochem. Soc. in press https://doi.org/10.1149/1945-7111/abe16d

\section{Manuscript version: Accepted Manuscript}

Accepted Manuscript is "the version of the article accepted for publication including all changes made as a result of the peer review process, and which may also include the addition to the article by IOP Publishing of a header, an article ID, a cover sheet and/or an 'Accepted Manuscript' watermark, but excluding any other editing, typesetting or other changes made by IOP Publishing and/or its licensors"

This Accepted Manuscript is @ 2021 The Author(s). Published by IOP Publishing Ltd..

As the Version of Record of this article is going to be/has been published on a gold open access basis under a CC 4.0 licence, this Accepted Manuscript is available for reuse under the applicable CC licence immediately.

Everyone is permitted to use all or part of the original content in this article, provided that they adhere to all the terms of the applicable licence referred to in the article - either https://creativecommons.org/licenses/by/4.0/ or https://creativecommons.org/licenses/by-nc-nd/4.0/

\begin{abstract}
Although reasonable endeavours have been taken to obtain all necessary permissions from third parties to include their copyrighted content within this article, their full citation and copyright line may not be present in this Accepted Manuscript version. Before using any content from this article, please refer to the Version of Record on IOPscience once published for full citation and copyright details, as permissions may be required. All third party content is fully copyright protected and is not published on a gold open access basis under a CC licence, unless that is specifically stated in the figure caption in the Version of Record.
\end{abstract}

View the article online for updates and enhancements. 


\section{A Modified Electrochemical Model to Account for Mechanical Effects Due to Lithium Intercalation and External Pressure}

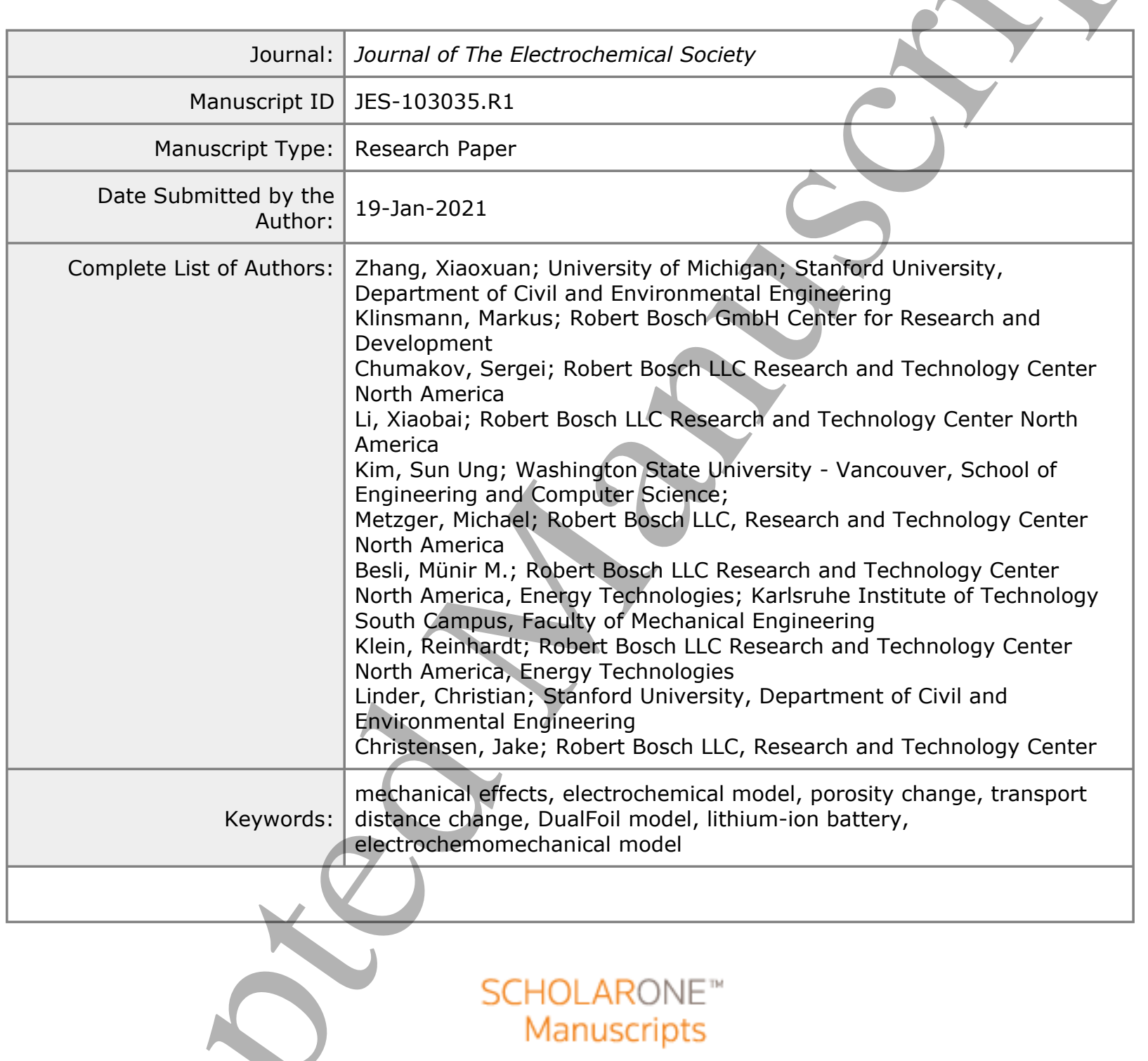




\title{
A modified electrochemical model to account for mechanical effects due to lithium intercalation and external pressure
}

\author{
Xiaoxuan Zhang ${ }^{1,2}$, Markus Klinsmann ${ }^{3}$, Sergei Chumakov ${ }^{4}$, Xiaobai Li $^{4}$, Sun Ung Kim ${ }^{4,5}$, \\ Michael Metzger ${ }^{4}$, Münir M. Besli ${ }^{4,6}$, Reinhardt Klein ${ }^{4}$, Christian Linder ${ }^{1,7, \dagger}$, Jake Christensen ${ }^{4, \ddagger}$ \\ ${ }^{1}$ Department of Civil and Environmental Engineering, Stanford University, Stanford, CA, 94305, USA \\ ${ }^{2}$ Department of Mechanical Engineering, University of Michigan, Ann Arbor, MI, 48109, USA \\ ${ }^{3}$ Corporate Research, Robert Bosch GmbH, D-71272 Renningen, Germany \\ ${ }^{4}$ Robert Bosch LLC, Research and Technology Center, Sunnyvale, CA, 94085, USA \\ ${ }^{5}$ School of Engineering and Computer Science, Washington State University, Vancouver, WA, 98686, USA \\ ${ }^{6}$ Department of Mechanical Engineering, Karlsruhe Institute of Technology (KIT), Karlsruhe 76131, Germany \\ ${ }^{7}$ Department of Mechanical Engineering, Stanford University, Stanford, CA, 94305, USA \\ Corresponding authors: ${ }^{\dagger}$ linder@
}

\begin{abstract}
For a battery cell, both the porosity of the electrodes/separator and the transport distance of charged species can evolve due to mechanical deformation arising from either lithium intercalation-induced swelling and contraction of the active particles or externally applied mechanical loading. To describe accurately the coupling between mechanical deformation and the cell's electrochemical response, we extend Newman's DualFoil model to allow variable, non-uniform porosities in both electrodes and the separator, which are dynamically updated based on the electrochemical and mechanical states of the battery cell. In addition, the finite deformation theory from continuum mechanics is used to modify the electrochemical transport equations to account for the change of the charged species transport distance. The proposed coupled electrochemomechanical model is tested with a parameterized commercial cell. Our simulation results confirm that mass conservation is satisfied with the new formulation. We further show that mechanical effects have a significant impact on the cell's electrochemical response at high charge/discharge rates.
\end{abstract}

Keywords mechanical effects $\cdot$ electrochemical model $\cdot$ porosity change $\cdot$ transport distance change $\cdot$ DualFoil model $\cdot$ lithium-ion battery $\cdot$ electrochemomechanical model

\section{Introduction}

Lithium-ion batteries (LIBs) are complex systems where electrochemistry, mechanics, and thermal phenomena are closely coupled [1-4]. Mechanical deformation can impact a battery cell's electrochemical response in several ways. For example, the energy density of a battery cell can be significantly improved by selecting silicon or germanium as the anode material [5, 6]. However, the diffusion-induced deformation is large enough to cause fracture of the active materials [7], leading to poor battery cyclic performance. For LIBs with conventional electrode materials, Li-intercalation causes the active materials to swell and contract, leading to a change of both the porosity of the electrodes and the species transport distance, which in turn affects the cell's electrochemical behavior [4, 8]. In addition, mechanical deformation can in some circumstances increase the driving force for Li-plating [9]. And mechanical abuse can trigger short circuit, causing catastrophic fáilure of battery cells [10]. Thus, understanding the impact of mechanical deformation on the cell's electrochemical response can provide a basis for innovations that ensure battery safety, increase cycle life, and improve performance (higher energy density, faster charge/discharge rate).

Newman's physics-based porous electrode model (the so-called DualFoil model) [11-14] has laid the foundation for modeling battery systems. Various extensions have been made in the past to account for thermal effects [15, 16] and other physical phenomena, such as side-reactions, film formation, and dendrite growth [17-22]. Though stress generation of active particles inside battery cells have been studied in [3, 4, 23, 24], the development of complete, coupled electrochemomechanical models for full cells is still scarce. Many existing mechanics-related modeling works are carried out only on the particle level [4, 23-29]. In a recent work [30], the authors solve a full set of electro-chemothermo-mechanical coupled equations in the three-dimensional (3D) space with the finite element method, which accounts for the change of both porosity and species transport distance. However, their model is computationally expensive to solve and would not be fast enough for optimization or exploring a large parameter space. 
In this work, a coupled electrochemomechanical model is proposed for battery cells made with conventional electrode materials in the one-dimensional (1D) setting. During charge/discharge, both the porosity of the electrodes/separator and the species transport distance evolves because of Li intercalation and the externally applied pressure, as illustrated in Fig. 1. In the new model, the porosity of each porous region is dynamically updated based on the electrochemical and mechanical state variables. The finite deformation theory from continuum mechanics is used to describe accurately the change of charged species transport distance. In addition, a modified version of the 4th order polynomial approximation for computing the concentration profile in the solid active material [31] is provided to account for the diffusion distance change due to Li-intercalation. In the proposed model, we do not account for the electrolyte movement, thus the total volume of electrolyte is assumed to be constant at each mesh location. It is worth noting that such assumption is valid only when the level of the externally applied mechanical loading is low.

\section{Model description}

In the original DualFoil model [11-14], a constant porosity is assumed for each porous region in a battery cell. However, such assumption is not accurate and does not account for volume changes caused by Li intercalation or externally applied mechanical loading, which can result in non-uniform porosity along the sandwich layer through direction (SLTD), as illustrated in Fig. 1] To account for mechanical effects, we need to distinguish the undeformed configuration and deformed configuration, which are also called the reference configuration and the current configuration in the continuum mechanics theory. We use upper case letters to denote terms defined in the reference configuration, and lower case for these defined in the current configuration. In the following, we first summarize the existing DualFoil model with a constant porosity. Following that, we discuss how to account for the change of the porosity and the species transport distance with a new set of equations.
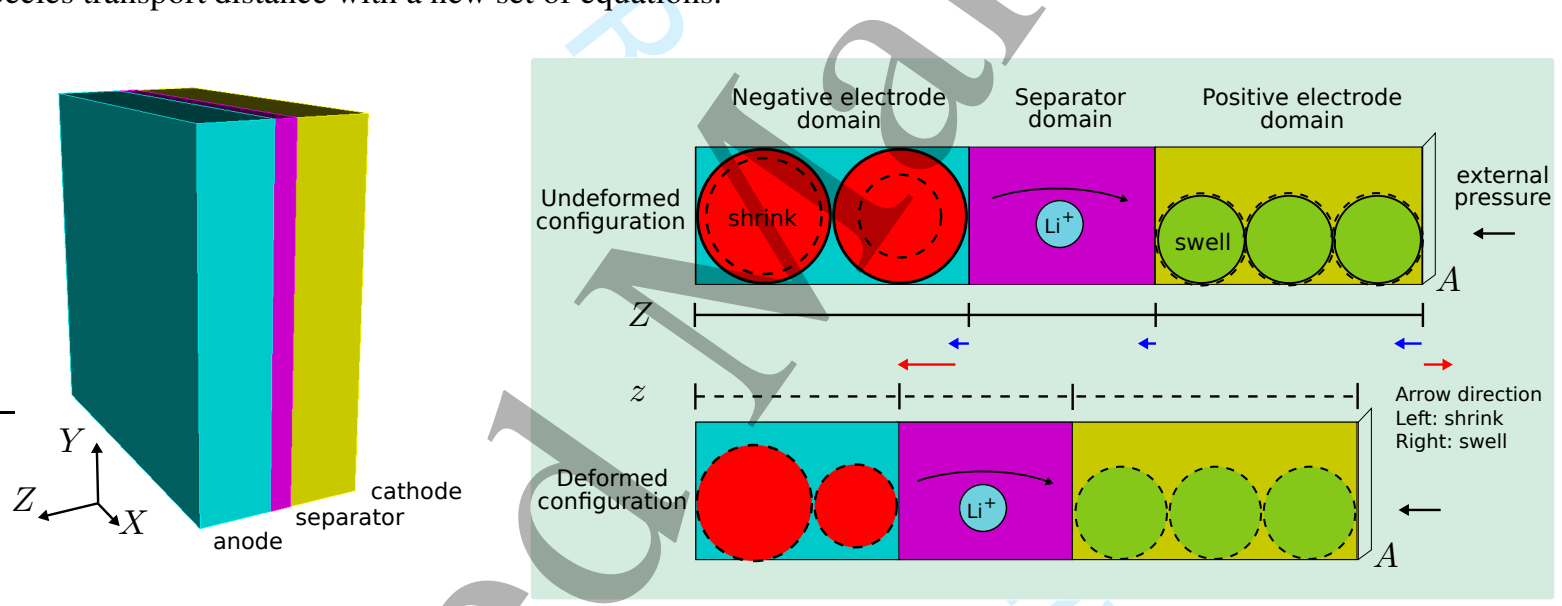

Figure 1: Illustration of a jellyroll sandwich layer in the 3D space (left) and the 1D modeling space for a discharge process (right). The letter $Z$ is used to represent the sandwich layer through direction (SLTD), which points from one electrode to the other. In the right figure, the solid line and the dashed line represent the undeformed (reference) configuration along $Z$ and the deformed (current) configuration along $z$, respectively. As the overall deformation in the X-Y plane is confined by the metal current collector and the cell case, we assume the averaged deformation only occurs in the $Z$ direction with a fixed cross-section area $A$ for both configurations 1 . During a discharge process, the volume contraction of graphite is larger than the volume swelling of $\mathrm{LiCoO}_{2}$, resulting in a total volume contraction as illustrated in the figure. The blue arrows indicate the modeling domain change due to external pressure, and red arrows for domain change due to Li insertion/removal. The actual length of the blue/red arrows for each porous region will be determined by the multiphysics model. Due to the volume change of the active particles, the negative electrode has an increased porosity, whereas the positive electrode has a decreased porosity. As shown in the deformed configuration, due to the spatial variation of $\mathrm{Li}$ concentration, the porosity across the electrode is non-uniform.

\subsection{Existing electrochemical model}

In conventional LIBs, two porous electrodes, which contain active energy storage materials, binders, and conductive additives, are separated by a porous separator, with the pores of all three regions being filled with liquid electrolyte

${ }^{1}$ This assumption is supported by the strain study with three-dimensional digital image correlation in [32], where the strain is found to be negligible in the $X$ and $Y$ directions compared to the $Z$ direction (the SLTD). 
Equation description

Equation

Electrolyte material balance

$\frac{\partial\left(\epsilon_{e}^{0} C_{e}\right)}{\partial t}=\nabla \cdot\left(D_{e, \mathrm{eff}} \nabla C_{e}+\frac{\boldsymbol{I}_{e}\left(1-t_{+}\right)}{F}\right)$

Electrolyte-phase Ohm's law $\boldsymbol{I}_{e}=-\kappa_{e, \mathrm{eff}} \nabla \phi_{e}+2 \frac{\kappa_{e, \mathrm{eff}} R \theta}{F}\left(1-t_{+}\right)\left(1+\frac{\partial \ln f_{ \pm}}{\partial \ln C_{e}}\right) \nabla \ln C_{e}$

Solid-phase Ohm's law

$\boldsymbol{I}_{s}=-\kappa_{s, \mathrm{eff}} \nabla \phi_{s}$

Charge conservation

$\nabla \cdot\left(\boldsymbol{I}_{s}+\boldsymbol{I}_{e}\right)=0 \quad$ with $\quad \nabla \cdot \boldsymbol{I}_{e}=-a_{0} F J_{n} \quad$ where $\quad a_{0}=\epsilon_{s}^{0} \frac{3}{R_{p}^{0}}$

Butler-Volmer insertion kinetics $J_{n}=k_{0} C_{s}^{\alpha_{c}} C_{e}^{\alpha_{a}}\left(C_{s}^{\max }-C_{s}\right)^{\alpha_{a}}\left[\exp \left(\frac{\alpha_{a} F}{R \theta} \eta_{s}\right)-\exp \left(-\frac{\alpha_{c} F}{R \theta} \eta_{s}\right)\right]$ with $\eta_{s}=\phi_{s}-\phi_{e}-U_{s}$

Intercalate material balance

$\frac{d}{d t} \bar{C}+3 \frac{J_{n}}{R_{p}^{0}}=0$

Volume-averaged flux relation $\frac{d}{d t} \bar{Q}+30 \frac{D_{s}}{\left(R_{p}^{0}\right)^{2}} \bar{Q}+\frac{45}{2} \frac{J_{n}}{\left(R_{p}^{0}\right)^{2}}=0$

Intercalate boundary conditions

Table 1: Summary of equations to describe the electrochemistry in the DualFoil model with constant porosity [33].

[33]. During charge/discharge cycles, Li ions travel from one electrode to the other through the electrolyte, whereas electrons are conducted between the electrodes through an external circuit. In this process, electrochemical reactions occur at the electrode-electrolyte interfaces. The applied current as well as the distribution of electronic versus ionic current causes the electric potential to vary in both the liquid electrolyte phase and the solid phase. Heat is generated due to irreversible resistive heating, reversible entropic heating, and chemical reactions [15]. The DualFoil model and its extended versions for LIBs are capable of describing these physical processes and computing the voltage response, potential profiles, concentration profiles, and the heat generate rate by utilizing the porous electrode and concentrated solution theories. The related equations are summarized in Table 1, which are solved with a Crank-Nicolson method in the $1 \mathrm{D}$ setting.

\subsection{Porosity change}

Porosity $\epsilon_{e}$ (or the volume fraction of the electrolyte) has a significant impact on the solution of equations listed in Table 11, as it is fully integrated into them through either its explicit appearance or effective quantities. The latter are computed based on the Bruggeman's relationship [34]

with $\alpha$ as the so-called Bruggeman's exponent.

$$
(\bullet)_{\mathrm{eff}}=(\bullet) \epsilon_{e}^{\alpha}
$$

Definition of porosity. To properly account for the porosity change and obtain an algebraic relation between the initial porosity $\epsilon_{e}^{0}$ and the current porosity $\epsilon_{e}$, we consider the volume change of different components in each porous region (i.e. the electrodes or the separator). In the reference configuration, $\epsilon_{e}^{0}$ is computed as

$$
\epsilon_{e}^{0}=\frac{V_{\text {electrolyte }}}{V_{\text {tot }}}=\frac{V_{\text {tot }}-V_{\text {add }}-V_{\text {film }}-V_{\text {active }}}{V_{\text {tot }}}=1-\frac{V_{\text {add }}}{V_{\text {tot }}}-\frac{V_{\text {film }}}{V_{\text {tot }}}-\frac{V_{\text {active }}}{V_{\text {tot }}}
$$

for each region, where the total volume $V_{\text {tot }}$ equals to the sum of the partial volumes of the electrolyte $V_{\text {electrolyte }}$, active materials $V_{\text {active }}$, additional components $V_{\text {add }}$, and the film $V_{\text {film }}$ produced by side reactions. Under the assumption that the liquid electrolyte is incompressible, similar to (2), the porosity $\epsilon_{e}$ in the current configuration is computed as

$$
\epsilon_{e}=\frac{v_{\text {electrolyte }}}{v_{\text {tot }}}=\frac{V_{\text {electrolyte }}}{v_{\text {tot }}}=\frac{V_{\text {tot }}}{v_{\text {tot }}}-\frac{V_{\text {add }}}{v_{\text {tot }}}-\frac{V_{\text {film }}}{v_{\text {tot }}}-\frac{V_{\text {active }}}{v_{\text {tot }}} .
$$


Here, $V$ denotes an initial volume defined in the reference configuration and $v$ is for a volume defined in the current configuration. In the separator, we have $V_{\text {add }}=V_{\text {film }}=v_{\text {add }}=v_{\text {film }}=0$.

Description of deformation. Since the reversible intercalation-induced volume change of a cell made with conventional electrode materials is relatively small (ca. 2.0\% [35] and ca. 3.3\% from our experiments), and only mild external mechanical loading is considered, the linear elastic theory is sufficient to describe the deformation and the volume change of each porous component. The displacement vector of each component of a cell is denoted as $\boldsymbol{u}(\boldsymbol{x}, t)$, which is a function of the coordinate $x$ and time $t$. The total strain tensor is defined as the symmetric part of the displacement gradient

$$
\varepsilon=\frac{1}{2}\left[\nabla \boldsymbol{u}+(\nabla \boldsymbol{u})^{T}\right]
$$

with the superscript $T$ denoting a transpose operation. The ratio of the total volume change is expressed through the trace of the total strain tensor $\varepsilon$ as

which results in the following relation

$$
\operatorname{tr}[\varepsilon]=\frac{\Delta v_{\text {tot }}}{V_{\text {tot }}}=\frac{v_{\text {tot }}-V_{\text {tot }}}{V_{\text {tot }}},
$$

$$
v_{\text {tot }}=(1+\operatorname{tr}[\varepsilon]) V_{\text {tot }},
$$

between the initial total volume $V_{\text {tot }}$ and the changed total volume $v_{\text {tot }}$. The entire volume change of each region is assumed to arise either due to elastic deformation from an externally applied mechanical loading or due to an internal volume change induced by Li-intercalation in active materials or the film growth, which has an additive relation

$$
\Delta v_{\text {tot }}=\Delta v_{\text {elastic }}+\Delta v_{\text {active }}+\Delta v_{\text {film }} \text {. }
$$

Thermal expansion induced volume change is neglected, as it is orders of magnitude smaller than these factors accounted in this work [36-38]. Within the scope of linear elastic theory, the strain tensor is expressed as

$$
\varepsilon=\varepsilon_{\text {elastic }}+\varepsilon_{\text {active }}+\varepsilon_{\text {film }}
$$

where $\varepsilon_{\text {elastic }}, \varepsilon_{\text {active }}$, and $\varepsilon_{\text {film }}$ represent the elastic strain from external mechanical loading, the intercalation induced strain from active materials, and the film growth induced strain, respectively. The insertion of (7) into (5) leads to

with

$$
\operatorname{tr}[\varepsilon]=\frac{\Delta v_{\text {elastic }}}{V_{\text {tot }}}+\frac{\Delta v_{\text {active }}}{V_{\text {tot }}}+\frac{\Delta v_{\text {film }}}{V_{\text {tot }}}
$$

$$
\operatorname{tr}\left[\varepsilon_{\text {elastic }}\right]=\frac{\Delta v_{\text {elastic }}}{V_{\text {tot }}}, \quad \operatorname{tr}\left[\varepsilon_{\text {active }}\right]=\frac{\Delta v_{\text {active }}}{V_{\text {tot }}}, \quad \text { and } \operatorname{tr}\left[\varepsilon_{\text {film }}\right]=\frac{\Delta v_{\text {film }}}{V_{\text {tot }}} .
$$

As the strain in the $X$ and $Y$ directions is found to be negligible compared to it in the SLTD [32], the strain tensor only has one non-zero diagonal term. Eqs. (5) and (10) can be simplified as

$$
\operatorname{tr}[\varepsilon]=\varepsilon^{z z}=\frac{\Delta v_{\text {tot }}}{V_{\text {tot }}}, \quad \operatorname{tr}\left[\varepsilon_{\text {elastic }}\right]=\varepsilon_{\text {elastio }}^{z z}=\frac{\Delta v_{\text {elastic }}}{V_{\text {tot }}}, \quad \operatorname{tr}\left[\varepsilon_{\text {active }}\right]=\varepsilon_{\text {active }}^{z z}=\frac{\Delta v_{\text {active }}}{V_{\text {tot }}}, \quad \operatorname{tr}\left[\varepsilon_{\text {film }}\right]=\varepsilon_{\text {film }}^{z z}=\frac{\Delta v_{\text {film }}}{V_{\text {tot }}},
$$

where the superscript $z$ denotes the SLTD, as illustrated in Fig. 1. In (11), $\Delta v_{\text {film }}$ is obtained by accounting for different side-reactions [19-21] in the electrochemical model. Though the proposed formulations account for porosity change due to film growth, we neglect the side reactions in this work for simplicity.

Intercalation induced deformation of active materials. For the active materials, the intercalation induced volume change is much larger than the elastic deformation caused by externally applied mechanical loading. For example, the volume change by $\mathrm{Li}$ insertion and extraction is ca. $10 \%$ for graphite [35, 39] and ca. $2 \%$ for $\mathrm{LiCoO}_{2}$ [40], whereas for an externally applied high stack/pressure of $5 \mathrm{MPa}$ [9], the resulting volumetric strain is ca. $0.01 \%$ for graphite (with a bulk modulus of $35.8 \mathrm{GPa}$ [41]) and ca. $0.002 \%$ for $\mathrm{LiCoO}_{2}$ (with a bulk modulus of $205.6 \mathrm{GPa}$ [42]). Thus, we can neglect the elastic deformation of the active material that results from the homogenized stress in the composite materials and express $v_{\text {active }}$ in the current configuration purely due to Li-interaction as

$$
v_{\text {active }}=V_{\text {active }}(1+\overline{\Omega(C)}) \text { with } \overline{\Omega(C)}=\frac{1}{\bar{V}} \int_{\mathcal{B}} \Omega(C(\boldsymbol{X})) d V
$$

where $\overline{\Omega(C)}$ is the average relative volume change ratio of the active material. In (12), Li concentration $C(\boldsymbol{X})$ is a location dependent quantity defined in the reference configuration, $\Omega(C)$ is the local relative volume change, $\bar{V}$ is the total volume of an active particle, and $\mathcal{B}$ is the domain describing the particle. In general, the intercalationinduced strain and Li concentration $C$ has a non-linear relation [40, 43], as shown in Fig. 5. If such a relation is linear, the average volume change can be computed directly from the volume averaged Li concentration $\bar{C}$ with 


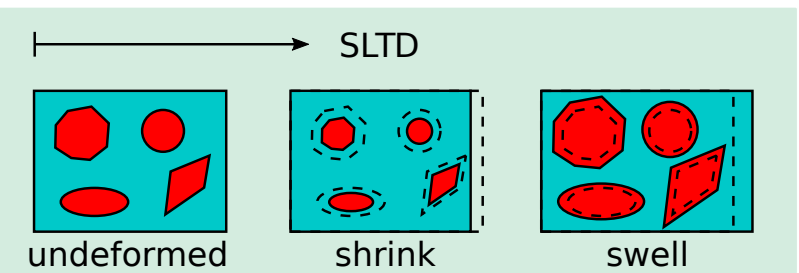

Figure 2: Illustration of the swelling/shrinkage of active particles in the 1D homogenized electrochemical model along the SLTD. Considering a representative 1D element with the given detailed microstructure in the undeformed configuration (only the active particle and the electrolyte are shown), the active particles could have different shapes and deform in any direction. Because the lateral deformation is confined by the case and metal current collector foils, the final deformation of the 1D element can only occur in the SLTD. As we do not account for the electrolyte movement across different elements, the total volume of the electrolyte is the same in the undeformed/shrink/swell configurations.

$\overline{\Omega(C)}=\Omega(\bar{C})=\omega\left(\bar{C}-\bar{C}^{0}\right)$, where $\omega$ is a swelling coefficient and $\bar{C}^{0}$ is the averaged initial Li concentration in the active particle. By inserting (12) into (11), we have

$$
\varepsilon_{\text {active }}^{z z}=\frac{\overline{\Omega(C)} V_{\text {active }}}{V_{\text {tot }}}
$$

Since the value of $\overline{\Omega(C)}$ depends on $C$, and the distribution of $C$ along the SLTD, in general, is non-uniform, $\varepsilon_{\text {active }}^{z z}$ is inhomogeneous along the SLTD.

Remark: In a battery cell, the active particles have different shapes (e.g. flake, spherical, etc.) that are not aligned along a specific direction, as shown in Fig. 2 The swelling/shrinkage of active particles could occur in any direction in the 3D space. When active particles deform in any direction, the particles will interact with the electrolyte. Since the lateral deformation is confined by the case and the metal current collector foils, the electrolyte can only flow in the SLTD. Thus, all the volume change of the active particles will result in a volume change of a porous region only in the SLTD due to the movement of the incompressible electrolyte. As we do not explicitly model electrolyte movement, the implication of the electrolyte movement in the neighborhood of a mesh point is thus reflected in Eq. (13) by stating that the volume change of the active particles causes a total volume change of a porous region only in the SLTD. Eq. (13) should not be interpreted as active particles are only allowed to deform in the SLTD.

Homogenized elastic deformation. The elastic strain tensor $\varepsilon_{\text {elastic }}$ describes the homogenized deformation of an entire composite layer (e.g., electrode or separator), rather than its individual components, because it is experimentally easier to measure the average deformation and mechanical properties of a porous region ${ }^{2}$. The non-zero component of the elastic strain tensor $\varepsilon_{\text {elastic }}$ is computed as

$$
\varepsilon_{\text {elastic }}^{z z}=\frac{1}{E} \sigma
$$

where $E$ and $\sigma$ are the effective Young's modulus and the homogenized Cauchy stress for the composites in the SLTD, respectively. Each porous region has a different $E$, which needs to be measured by experiments. Thus, the resulting $\varepsilon_{\text {elastic }}^{z z}$ is the same inside each region, but is different among regions.

Updated porosity. Now, the porosity of electrode regions in the current configuration given in (3) can be rewritten as

$$
\epsilon_{e}=\frac{1-\epsilon_{\mathrm{add}}^{0}-\epsilon_{\mathrm{active}}^{0}}{1+\operatorname{tr}[\varepsilon]}=\frac{\epsilon_{\mathrm{e}}^{0}}{1+\operatorname{tr}[\varepsilon]}
$$

with $\epsilon_{\text {active }}^{0}$ and $\epsilon_{\text {add }}^{0}$ as the initial volume fraction for the active materials and the additive materials, respectively, which are computed as

$$
\epsilon_{\text {active }}^{0}=\frac{V_{\text {active }}}{V_{\text {tot }}} \quad \text { and } \quad \epsilon_{\text {add }}^{0}=\frac{V_{\text {add }}}{V_{\text {tot }}} .
$$

For the porous separator, which does not contain the active materials, Eq. (15) can be simplified as

$$
\epsilon_{e}=\frac{1-\epsilon_{\mathrm{solid}}^{0}}{1+\operatorname{tr}[\varepsilon]}=\frac{\epsilon_{\mathrm{e}}^{0}}{1+\operatorname{tr}[\varepsilon]}
$$

${ }^{2}$ For each porous region, as the electrolyte is incompressible, and the elastic deformation of the active materials caused by externally applied stress is negligible, the homogenized elastic strain $\varepsilon_{\text {elastic }}$ is mainly attributed to the compressible additive materials in the electrodes and the solid phase in the separator, and potentially any unfilled pores in these regions. 
A PREPRINT - JANUARY 19, 2021

with $\epsilon_{\text {solid }}^{0}$ as the volume fraction of the solid phase materials. The strain tensor $\varepsilon$ for the separator only contains the elastic component $\varepsilon_{\text {elastic }}$ with $\operatorname{tr}\left[\varepsilon_{\text {film }}\right]=0$ and $\operatorname{tr}\left[\varepsilon_{\text {active }}\right]=0$.

\subsection{Modified electrochemical formulations}

In addition to the porosity change, mechanical deformation impacts the solution of the equations listed in Table 1 via changing the domain over which these equations are solved, as illustrated in Fig. 11 To ensure the equivalence of equations that describe physics in both the reference configuration and the deformed configuration, we follow the continuum mechanics theory, which ensures consistency of physical properties of a continuum defined in arbitrary coordinate systems. Terms to account for the effect of deformation will appear in the relevant equations. We start with a finite strain formulation and define the mapping of physical properties to different configurations. To make the result more accessible to readers without a mechanics background, we apply the approximation of linear elastic theory to derive formulations that resemble those in Table 1

Coordinate transformation. The finite strain formulation requires the differentiation between upper- and lower-case symbols, with the former describing quantities defined in the reference configuration and the latter those in the current configuration. The displacement $\boldsymbol{u}$ is defined as $\boldsymbol{u}=\boldsymbol{x}-\boldsymbol{X}$, with $\boldsymbol{x}$ and $\boldsymbol{X}$ describing the current coordinate and the reference (initial) coordinate of the same part of a body, respectively. The deformation gradient $\boldsymbol{F}$ is defined as

$$
\boldsymbol{F}=\frac{\partial \boldsymbol{x}}{\partial \boldsymbol{X}}=\mathbf{1}+\nabla_{\boldsymbol{X}} \boldsymbol{u}
$$

where 1 is the second-order identity tensor. For the problem considered in this work, where the in-plane strain is negligible and the cell behavior is described with the 1D model in the SLTD, the deformation gradient $\boldsymbol{F}$ has the form

$$
\boldsymbol{F}=\left[\begin{array}{ccc}
1 & 0 & 0 \\
0 & 1 & 0 \\
0 & 0 & 1+\frac{\partial u_{3}}{\partial X_{3}}
\end{array}\right] \boldsymbol{e}_{i} \otimes \boldsymbol{E}_{J}
$$

with $\boldsymbol{e}_{i}$ and $\boldsymbol{E}_{J}$ representing the basis vectors in the current and reference configurations, respectively. The term $\frac{\partial u_{3}}{\partial X_{3}}$ is equivalent to $\varepsilon^{z z}$ in 11. A volume $d v$ defined in the current configuration is related to its equivalent representation $d V$ in the reference configuration with

$$
d v=\mathcal{J} d V \quad \text { with } \mathcal{J}=\operatorname{det}[\boldsymbol{F}]
$$

where $\operatorname{det}[\boldsymbol{F}]$ is called Jacobian (equivalent to $1+\operatorname{tr}[\varepsilon]$ in (5) for the linear elastic theory) to describe the volume change ratio.

For a quantity defined per unit volume, such as concentration, we ensure the equivalent description by enforcing

$$
c d v=C d V
$$

which leads to the relation $c=C / \mathcal{J}$ with $c$ and $C$ as the concentration in the current and reference configuration, respectively. The reference gradient $\nabla_{\boldsymbol{X}}$ of a quantity $(\bullet)$ is transformed to its spatial gradient $\nabla_{\boldsymbol{x}}$ by

$$
\nabla_{\boldsymbol{x}}(\bullet)=\boldsymbol{F}^{-T} \nabla_{\boldsymbol{X}}(\bullet)
$$

An area element $d \boldsymbol{a}$ in the current configuration is related to its reference representation $d \boldsymbol{A}$ through

$$
d \boldsymbol{a}=\mathcal{J} \boldsymbol{F}^{-T} d \boldsymbol{A} .
$$

To ensure that the rates of particles flowing through an area in two different configurations are identical, we have $\boldsymbol{k} \cdot d \boldsymbol{a}=\boldsymbol{K} \cdot d \boldsymbol{A}$, resulting in

$$
\boldsymbol{k}=\mathcal{J}^{-1} \boldsymbol{F} \boldsymbol{K}
$$

with $\boldsymbol{k}$ and $\boldsymbol{K}$ as the flux defined in current and reference configuration, respectively. Note that any flux variable (e.g., current density, which is a flux of charge) obeys the same relationship.

Electrolyte material balance. The electrolyte material balance in the reference configuration has the form

$$
\frac{\partial\left(\epsilon_{e}^{0} C_{e}\right)}{\partial t}=-\nabla_{\boldsymbol{X}} \cdot(\boldsymbol{Q})+\nabla_{\boldsymbol{X}} \cdot\left(\frac{\boldsymbol{I}_{e}\left(1-t_{+}\right)}{F}\right)
$$

where $\boldsymbol{Q}$ is the Li-ion flux due to the concentration gradient and $\boldsymbol{I}_{e}$ is the current density in the reference configuration. The spatial flux $\boldsymbol{q}$ is computed as

$$
\boldsymbol{q}=-D_{e, \mathrm{eff}} \nabla_{\boldsymbol{x}}\left(c_{e}\right),
$$


where the effective electrolyte diffusivity $D_{e, \text { eff }}$ is computed based on 11) with the updated porosity. Assuming the liquid electrolyte is incompressible, we have $\mathcal{J}_{e}=1$, resulting in $c_{e}=C_{e}$. The readers should notice the difference between $\mathcal{J}_{e}$ and $\mathcal{J}$, where the former describes the volume change of the liquid electrolyte, and the latter is the total volume change of a porous region. Based on (22), (24), and (26), $Q$ is computed as

$$
\boldsymbol{Q}=\mathcal{J} \boldsymbol{F}^{-1} \boldsymbol{q}=-D_{e, \mathrm{eff}} \mathcal{J} \boldsymbol{F}^{-1} \boldsymbol{F}^{-T} \nabla_{\boldsymbol{X}}\left(C_{e}\right)
$$

Inserting 27) into (25) yields the final form for the electrolyte mass balance equation as

$$
\frac{\partial\left(\epsilon_{e}^{0} C_{e}\right)}{\partial t}=\nabla_{\boldsymbol{X}} \cdot\left(D_{e, \mathrm{eff}} \mathcal{J} \boldsymbol{F}^{-1} \boldsymbol{F}^{-T} \nabla_{\boldsymbol{X}}\left(C_{e}\right)\right)+\nabla_{\boldsymbol{X}} \cdot\left(\frac{\boldsymbol{I}_{e}\left(1-t_{+}\right)}{F}\right)
$$

Electrolyte-phase Ohm's law. The electrolyte current density $\boldsymbol{i}_{e}$ in the current configuration is computed as

$$
\boldsymbol{i}_{e}=-\kappa_{e, \mathrm{eff}} \nabla_{\boldsymbol{x}} \phi_{e}+2 \frac{\kappa_{e, \mathrm{eff}} R \theta}{F}\left(1-t_{+}\right)\left(1+\frac{\partial \ln f_{ \pm}}{\partial \ln c_{e}}\right) \nabla_{\boldsymbol{x}} \ln c_{e},
$$

where the effective electrolyte conductivity $\kappa_{e, \text { eff }}$ is computed based on (1) with the updated porosity. Based on (22) and (24), the reference current density $\boldsymbol{I}_{e}$ is computed as

$$
\boldsymbol{I}_{e}=\mathcal{J} \boldsymbol{F}^{-1} \boldsymbol{F}^{-T}\left(-\kappa_{e, \mathrm{eff}} \nabla_{\boldsymbol{X}} \phi_{e}+2 \frac{\kappa_{e, \mathrm{eff}} R \theta}{F}\left(1-t_{+}\right)\left(1+\frac{\partial \ln f_{ \pm}}{\partial \ln C_{e}}\right) \nabla_{\boldsymbol{X}} \ln C_{e}\right)
$$

Solid-phase Ohm's law. The solid phase current density $\boldsymbol{i}_{s}$ in the current configuration is computed as

$$
i_{s}=-\kappa_{s, \text { eff }} \nabla_{\boldsymbol{x}} \phi_{s}
$$

Based on (22) and (24), we obtain the reference current density

$$
\boldsymbol{I}_{s}=\mathcal{J} \boldsymbol{F}^{-1} \boldsymbol{F}^{-T}\left(-\kappa_{s, \text { eff }} \nabla_{\boldsymbol{X}} \phi_{s}\right)
$$

Butler-Volmer insertion kinetics. For a spherical particle, we use $\boldsymbol{j}$ and $\boldsymbol{J}$ to denote the Li-ion surface fluxes in the current and reference configuration, $\boldsymbol{n}$ and $\boldsymbol{N}$ to denote the surface normal associated to $\boldsymbol{j}$ and $\boldsymbol{J}$, respectively. The normal flux of Li ions on a particle's surface in the current configuration is expressed as

$$
j_{n}=\boldsymbol{j} \cdot \boldsymbol{n}=k_{0} c_{s}^{\alpha_{c}} c_{e}^{\alpha_{a}}\left(c_{s}^{\max }-c_{s}\right)^{\alpha_{a}}\left[\exp \left(\frac{\alpha_{a} F}{R \theta} \eta_{s}\right)-\exp \left(-\frac{\alpha_{c} F}{R \theta} \eta_{s}\right)\right]
$$

Under the assumption of isotropic deformation, we have

$$
\overline{\boldsymbol{F}}_{\text {active }}=\overline{\mathcal{J}}_{\text {active }}^{1 / 3} \mathbf{1}, \quad \overline{\boldsymbol{F}}_{\text {active }}^{-1}=\overline{\mathcal{J}}_{\text {active }}^{-1 / 3} \mathbf{1}, \quad \text { and } \quad \boldsymbol{n}=\boldsymbol{N}
$$

for a spherical particle, where $\overline{\boldsymbol{F}}_{\text {active }}$ is the deformation gradient to represent the overall deformation of the whole particle rather than any specific location, and $\overline{\mathcal{J}}_{\text {active }}$ is the averaged volume change ratio of the particle with $\overline{\mathcal{J}}_{\text {active }}=$ $1+\overline{\Omega(C)}$. Based on (24), the reference flux $\boldsymbol{J}$ is computed as

$$
\boldsymbol{J}=\overline{\mathcal{J}}_{\text {active }} \overline{\boldsymbol{F}}_{\text {active }}^{-1} \boldsymbol{j}=\overline{\mathcal{J}}_{\text {active }}^{2 / 3} \boldsymbol{j}
$$

The normal flux of Li ions on a particle's surface in the reference configuration is thus expressed as

$$
J_{n}=\boldsymbol{J} \cdot \boldsymbol{N}=\overline{\mathcal{J}}_{\text {active }}^{2 / 3} \boldsymbol{j} \cdot \boldsymbol{n}=\overline{\mathcal{J}}_{\text {active }}^{2 / 3} j_{n}
$$

With $c_{s}=C_{s} / \overline{\mathcal{J}}_{\text {active }}, c_{e}=C_{e}$, and $\alpha_{a}=1-\alpha_{c}$, inserting (33) into (36) yields 3

$$
J_{n}=\overline{\mathcal{J}}_{\text {active }}^{-1 / 3}\left(k_{0}\left(C_{s}\right)^{\alpha_{c}} C_{e}^{\alpha_{a}}\left(C_{s}^{\max }-C_{s}\right)^{\alpha_{a}}\left[\exp \left(\frac{\alpha_{a} F}{R \theta} \eta_{s}\right)-\exp \left(-\frac{\alpha_{c} F}{R \theta} \eta_{s}\right)\right]\right) .
$$

Charge conservation. The charge balance equation in the reference configuration is expressed as

$$
\nabla_{\boldsymbol{X}} \cdot\left(\boldsymbol{I}_{s}+\boldsymbol{I}_{e}\right)=0 \text { with } \nabla_{\boldsymbol{X}} \cdot \boldsymbol{I}_{e}=-a_{0} F J_{n}
$$

where $a_{0}=3 \epsilon_{s}^{0} / R_{p}^{0}[44]$.

${ }^{3}$ Note that this relationship is more complex when we relax the assumption that $\alpha_{a}+\alpha_{c}=1$. 
A PREPRINT - JANUARY 19, 2021

Mass balance in active particles. In the reference configuration, the mass diffusion in solids is governed by

$$
\frac{\partial C_{s}}{\partial t}+\nabla_{X} \cdot \boldsymbol{Q}=0
$$

where $Q$ is the molar flux, which in general is composition and pressure dependent [4]. As shown in [4], the composition distribution is not significantly influenced by pressure except at high dimensionless current, therefore we neglect the pressure dependence of $\boldsymbol{Q}$ in the present model. We only consider a composition dependent $\boldsymbol{Q}$ governed by (in the current configuration)

$$
\boldsymbol{q}=-D_{s} \nabla_{\boldsymbol{x}}\left(c_{s}\right)
$$

Based on (22) and (24), Eq. (39) becomes

$$
\frac{\partial C_{s}}{\partial t}-\nabla_{\boldsymbol{X}} \cdot\left(D_{s} \mathcal{J}_{\text {active }} \boldsymbol{F}_{\text {active }}^{-1} \boldsymbol{F}_{\text {active }}^{-T} \nabla_{\boldsymbol{X}}\left(\frac{C_{s}}{\mathcal{J}_{\text {active }}}\right)\right)=0
$$

where $\boldsymbol{F}_{\text {active }}$ is the deformation gradient at a specific location inside the particle, and $\mathcal{J}_{\text {active }}$ is the corresponding volume change ratio at that location, which are location dependent. We assume that the active particle is disordered with lithium diffusion and lattice expansion/contraction being isotropic [4, 23]. Eq. (41) becomes

$$
\frac{\partial C_{s}}{\partial t}-\nabla_{\boldsymbol{X}} \cdot\left(D_{s} \mathcal{J}_{\text {active }}^{1 / 3} \nabla_{\boldsymbol{X}}\left(\frac{C_{s}}{\mathcal{J}_{\text {active }}}\right)\right)=0
$$

with $\boldsymbol{F}_{\text {active }}=\mathcal{J}_{\text {active }}{ }^{1 / 3} 1$. Eq. (42) can be solved by one extra discretization in the particle radial direction in addition to the main discretization in the SLTD (so-called pseudo 2D approach). Alternatively, one can utilize the three-parameter polynomial approximation presented in [31], where no additional discretization is needed, and the computational cost can be reduced significantly. Note that the formulation in [31] must be modified further, as in [45], to handle concentration-dependent diffusivities. For simplicity, the present model is restricted to concentrationindependent solid-phase diffusivity. Even in this case, the formulation in [31] is not directly applicable to [42], because $\mathcal{J}_{\text {active }}$ is a radial location dependent quantity. Thus, to facilitate the computationally efficient polynomial approach, we further approximate $\mathcal{J}_{\text {active }}$ with its volume averaged value $\overline{\mathcal{J}}_{\text {active }}$, which has no location dependence, to simplify (42) into

$$
\frac{\partial C_{s}}{\partial t}-\nabla_{\boldsymbol{X}} \cdot\left(\frac{D_{s}}{\overline{\mathcal{J}}_{\text {Jactive }}^{2 / 3}} \nabla_{\boldsymbol{X}}\left(C_{s}\right)\right)=0
$$

or with the form written in the spherical coordinate system as

$$
\frac{\partial C_{s}}{\partial t}-\frac{D_{s}}{\overline{\mathcal{J}}_{\text {active }}^{2 / 3}} \frac{1}{R^{2}} \frac{\partial}{\partial R}\left(R^{2} \frac{\partial C_{s}}{\partial R}\right)=0 .
$$

Eq. (44) is solved with a slightly modified version (see (51)-(53) in Table 2) of the formulation presented in [31]. A comparison study of the surface concentration $C_{s}$ resulted from (42) and its simplified form (43) with finite element methods in a spherical particle is given in the results section to show the accuracy of (43). Our simulation results confirm that the total amount of $\mathrm{Li}$ in both electrode active materials and the electrolyte is conserved with the new formulations.

1D and small deformation simplification. In the 1D setting under the assumption of small deformation, we have

$$
\boldsymbol{F}=1+\varepsilon^{z z}, \boldsymbol{F}^{-1}=\frac{1}{1+\varepsilon^{z z}}, \boldsymbol{F}^{-T}=\frac{1}{1+\varepsilon^{z z}}, \mathcal{J}=1+\varepsilon^{z z}, \overline{\mathcal{J}}_{\text {active }}=1+\frac{\varepsilon_{\text {active }}^{z z}}{\epsilon_{\text {active }}^{0}}
$$

which lead to the simplification shown in Eqs. (46)-(50) of Table 2

The proposed coupled electrochemomechanical model is solved with a Crank-Nicolson method in the 1D setting. The same BCs as used in [12, 13] are applied to Eqs. (46)-(53) to form a well-posed problem. This new model takes the homogenized mechanical stress $\sigma$ in the SLTD as an input to update the porosity embedded in Eqs. (46)-(53). The homogenized stress $\sigma$ can be obtained by solving the linear momentum balance equation with an appropriate numerical tool, such as finite element methods, which is out of the scope of this work. In the results section, the externally applied stress value is pre-defined as a time-invariant boundary condition. To fully utilize the capability of the proposed model, a coupling between the electrochemical model with a mechanical or thermomechanical solver is needed to dynamically update $\sigma$ based on the cell electrochemical states, which will be the subject of a subsequent paper [46]. 
Equation description

Equation

Electrolyte material balance

$\frac{\partial\left(\epsilon_{e}^{0} C_{e}\right)}{\partial t}=\nabla_{X} \cdot\left(\frac{D_{e, \text { eff }}}{1+\varepsilon^{z z}} \nabla_{X}\left(C_{e}\right)\right)+\nabla_{X} \cdot\left(\frac{\boldsymbol{I}_{e}\left(1-t_{+}\right)}{F}\right)$

Electrolyte-phase Ohm's law $\boldsymbol{I}_{e}=\frac{-\kappa_{e, \mathrm{eff}} \nabla_{X} \phi_{e}+2 \frac{\kappa_{e, \mathrm{eff}} R \theta}{F}\left(1-t_{+}\right)\left(1+\frac{\partial \ln f_{ \pm}}{\partial \ln C_{e}}\right) \nabla_{X} \ln C_{e}}{1+\varepsilon^{z z}}$

Solid-phase Ohm's law

$$
\boldsymbol{I}_{s}=\frac{1}{1+\varepsilon^{z z}}\left(-\kappa_{s, \mathrm{eff}} \nabla_{X} \phi_{s}\right)
$$

Charge conservation

Butler-Volmer insertion kinetics

$$
\nabla_{X} \cdot\left(\boldsymbol{I}_{s}+\boldsymbol{I}_{e}\right)=0 \text { with } \nabla_{X} \cdot \boldsymbol{I}_{e}=-a_{0} F J_{n} \text { where } a_{0}=\frac{3 \epsilon_{s}^{0}}{R_{p}^{0}}
$$

$$
J_{n}=\frac{k_{0} C_{s}^{\alpha_{c}} C_{e}^{\alpha_{a}}\left(C_{s}^{\max }-C_{s}\right)^{\alpha_{a}}\left[\exp \left(\frac{\alpha_{a} F}{R \theta} \eta_{s}\right)-\exp \left(-\frac{\alpha_{c} F}{R \theta} \eta_{s}\right)\right]}{\left(\overline{\mathcal{J}}_{\text {active }}\right)^{1 / 3}}
$$

Intercalate material balance

$$
\begin{gathered}
\frac{d}{d t} \bar{C}_{s}+3 \frac{J_{n}}{R_{p}^{0}}=0 \\
\frac{d}{d t} \bar{Q}+30 \frac{D_{s}}{\overline{\mathcal{J}}_{\text {active }}^{2 / 3}\left(R_{p}^{0}\right)^{2}} \bar{Q}+\frac{45}{2} \frac{J_{n}}{\left(R_{p}^{0}\right)^{2}}=0 \\
35 \frac{D_{s}}{\overline{\mathcal{J}}_{\text {active }}^{2 / 3} R_{p}}\left(C_{s}-\bar{C}\right)-8 \frac{D_{s}}{\overline{\mathcal{J}}_{\text {active }}^{2 / 3}} \bar{Q}=-J_{n}
\end{gathered}
$$

Volume-averaged flux relation

Intercalate boundary conditions

Table 2: Summary of new equations in the coupled electrochemomechanical model.
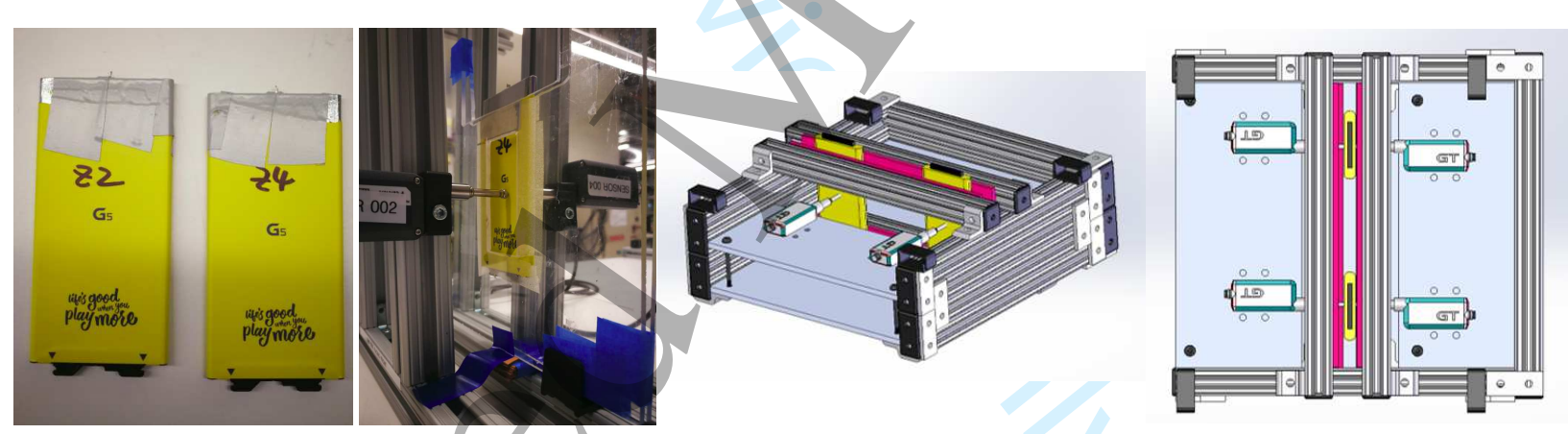

Figure 3: Illustration of the experiment setup. From left to right: the tested LG G5 cell phone battery with control circuit removed, the actual customized platform to measure the swelling of the cell with two visible displacement sensors, two CAD views of the experiment platform.

\section{Experiments and model parameterization}

We measured the electrochemical and mechanical behavior of several LG G5 cell phone batteries, purchased from the official website [71] with model number BL-42D1F. After removing the control circuit, the cells were mounted into a customized platform, as shown in Fig. 3. The structure of the platform was designed by following [72] with two highprecision displacement sensors ( $1 \mu \mathrm{m}$ accuracy and $0.1 \mu \mathrm{m}$ resolution, model number Keyence GT2-H12KL, Japan) mounted in opposite directions to measure the amount of swelling during charge/discharge. The entire platform with the specimen was placed into a Yamato convection oven (Model DKN400) under forced convective cooling at a temperature of $25{ }^{\circ} \mathrm{C}$. Several cells were charged/discharged at various $\mathrm{C}$-rates $(\mathrm{C} / 10, \mathrm{C} / 5, \mathrm{C} / 3, \mathrm{C} / 2, \mathrm{C} / 1) \mathrm{for}$ three times at each $\mathrm{C}$-rate, while their voltage and thickness changes were measured. For each $\mathrm{C}$-rate, the cells were discharged to $2.7 \mathrm{~V}$. Then, the cells were rest for $10 \mathrm{mins}$ before charge. At the end of each charge, to $4.4 \mathrm{~V}$, the voltage was held at $4.4 \mathrm{~V}$ until the current dropped to a C-rate of $\mathrm{C} / 20$. The cells were rest for 10 mins before discharge.

The cells were parameterized by combining reported values from the literature, measured values from experiments, and optimized values from simulations. During the parameterization procedure, one cell was opened in a glove box 
A PREPRINT - JANUARY 19, 2021

\begin{tabular}{|c|c|c|c|}
\hline $\mathrm{Li}_{\mathrm{x}} \mathrm{C}_{6}$ parameters & Value & Bound & Ref. \\
\hline $\begin{array}{l}\text { Thickness }^{a}, \mu \mathrm{m} \\
\text { Electrolyte volume fraction }^{b} \\
\text { Active material volume fraction } \\
\text { Diffusivity in solids }{ }^{b}, \mathrm{~m}^{2} / \mathrm{s} \\
\text { Particle radius }{ }^{b}, \mu \mathrm{m} \\
\text { Reaction rate constant }{ }^{b}, \mathrm{~m}^{2} / \mathrm{s} \cdot(\mathrm{m} / \mathrm{mol})^{1 / 2} \\
\text { Active material density, } \mathrm{g} / \mathrm{cm}^{3} \\
\text { Theoretical specific capacity }, b \mathrm{mAh} / \mathrm{g} \\
\text { Initial value of } \mathrm{x}^{, b} \\
\text { Matrix conductivity, } \mathrm{S} / \mathrm{m} \\
\text { Bruggeman exponent } \\
\text { Effective Young's modulus, } \mathrm{N} / \mathrm{m}^{2}\end{array}$ & $\begin{array}{l}79.4 \\
0.28 \\
0.659 \\
2.6 \mathrm{e}-14 \\
7.3 \\
7.2 \mathrm{e}-12 \\
2.26 \\
372 \\
0.871 \\
10 \\
1.3 \\
4.6 \mathrm{e} 8\end{array}$ & $\begin{array}{l}{[0.21,0.37]} \\
{[0.54,0.7]} \\
{[1 \mathrm{e}-15,1 \mathrm{e}-11]} \\
{[5,20]} \\
{[1.0 \mathrm{e}-12,1.0 \mathrm{e}-9]} \\
{[0.75,0.95]} \\
{[1,3.1]}\end{array}$ & $\begin{array}{l}{[33,47,48]} \\
{[47,49]} \\
{[43,50-52]} \\
{[33,49,53]} \\
{[33,54,55]} \\
{[33]}\end{array}$ \\
\hline $\mathrm{Li}_{\mathrm{y}} \mathrm{CoO}_{2}$ parameters & & & \\
\hline $\begin{array}{l}\text { Thickness }^{a}, \mu \mathrm{m} \\
\text { Electrolyte volume fraction }^{b} \\
\text { Active material volume fraction } \\
\text { Diffusivity in solids }{ }^{b}, \mathrm{~m}^{2} / \mathrm{s} \\
\text { Particle radius }{ }^{b}, \mu \mathrm{m} \\
\text { Reaction rate constant }{ }^{b}, \mathrm{~m}^{2} / \mathrm{s} \cdot(\mathrm{m} / \mathrm{mol})^{1 / 2} \\
\text { Active material density, } \mathrm{g} / \mathrm{cm}^{3} \\
\text { Theoretical specific capacity, }, \mathrm{mAh} / \mathrm{g} \\
\text { Initial value of y, } \\
\text { Matrix conductivity, S/m } \\
\text { Bruggeman exponent } \\
\text { Effective Young's modulus, } \mathrm{N} / \mathrm{m}^{2}\end{array}$ & $\begin{array}{l}54.6 \\
0.22 \\
0.72 \\
2.0 \mathrm{e}-13 \\
5.6 \\
1.4 \mathrm{e}-11 \\
5.10 \\
273.85 \\
0.353 \\
10\end{array}$ & $\begin{array}{l}{[0.15,0.35]} \\
{[0.55,0.80]} \\
{[1 \mathrm{e}-16,1 \mathrm{e}-11]} \\
{[3,9]} \\
{[1.0 \mathrm{e}-12,1.0 \mathrm{e}-9]} \\
{[0.33,0.5]} \\
{[1,3.0]}\end{array}$ & $\begin{array}{l}{[33,47,49]} \\
{[33,47,49]} \\
{[58} \\
{[33,65]} \\
{[33,64,67]} \\
{[33]} \\
{[33]} \\
{[33,56,68]} \\
{[57]}\end{array}$ \\
\hline Separator parameters & & & \\
\hline $\begin{array}{l}\text { Thickness }^{a}, \mu \mathrm{m} \\
\text { Electrolyte volume fraction }^{b} \\
\text { Bruggeman exponent } \\
\text { Effective Young's modulus, } \mathrm{N} / \mathrm{m}^{2}\end{array}$ & $\begin{array}{l}13.6 \\
0.4 \\
1.4 \\
5.0 \mathrm{e} 8 \\
\end{array}$ & $\begin{array}{l}{[0.35,0.55]} \\
{[1,3.0]}\end{array}$ & $\begin{array}{l}{[33,69,70]} \\
{[33,68]} \\
{[57]}\end{array}$ \\
\hline
\end{tabular}

Table 3: List of simulation parameters for electrodes and separator. The bounds of fitting parameters are chosen based either fitted or measured values given in the references.

to measure its design parameters, such as electrode thickness, separator thickness, and separator area. A number of $\mathrm{Li} / \mathrm{LiCoO}_{2}$ and $\mathrm{Li} /$ graphite coin cells were made with electrode materials harvested from fresh LGG5 cells to measure the electrode open-circuit potentials (OCPs), as shown in Fig. 4] Interested readers are directed to Ref [62] for a comparison of OCP measurements from the Galvanostatic intermittent titration technique (GITT) and the averaged voltage method for a LCO/graphite cell that is similar to the one studied in this work. The functional description of the electrolyte from [33] was used for our simulation, with their formulations/values summarized in Table 4 The mechanical properties for each porous region were chosen from [57]. For graphite, a measured non-linear volume change ratio for a different pouch cell, as shown in Fig. 5 , is used for the simulation, where $\overline{\Omega(C)}$ is computed based on (12). A linear swelling ratio of $\omega=1.8 \%$ is assumed for $\mathrm{LiCoO}_{2}$ [40] with $\overline{\Omega(C)}$ being computed based on $\overline{\Omega(C)}=\omega\left(\bar{C}-\bar{C}^{0}\right)$. The remaining parameters that could not be found in either of the sources mentioned, e.g., porosity, diffusion coefficient, reaction rate, etc., were determined by numerical optimization, i.e., by minimizing the L2-norm of the difference of the experimental and simulated voltage profile at given C-rates. The parameters used in the results section are summarized in Table 3 , 4 


\begin{tabular}{|c|c|}
\hline Parameter/Property & Value/Expression \\
\hline $\begin{array}{l}\text { Salt concentration }\left(1 \mathrm{M} \mathrm{LiPF}_{6} \text { in }\right. \\
\text { EC/DEC), M }\end{array}$ & 1.0 \\
\hline Conductivity, $\mathrm{mS} / \mathrm{cm}$ & $\begin{aligned} \kappa_{e, \mathrm{ref}} & =0.91 c_{e}\left[\left(-10.5+0.072 \theta-6.5 \times 10^{-5} \theta^{2}\right)\right. \\
& +\left(0.668-0.0172 \theta+2.6 \times 10^{-5} \theta^{2}\right) c_{e} \\
& \left.+\left(0.494-8.56 \times 10^{-4} \theta\right) c_{e}^{2}\right]^{2}\end{aligned}$ \\
\hline Diffusivity, $\mathrm{cm}^{2} / \mathrm{s}$ & $\begin{array}{l}\log _{10}\left\{D_{e} \exp \left[400\left(\frac{1}{\theta}-\frac{1}{298}\right)\right]\right\} \\
=-5.08-0.22 c_{e}-\frac{55.87}{\theta-227-517 c}\end{array}$ \\
\hline Transference number & $t_{+}=0.399 \quad \theta-23 r-5.16$ \\
\hline Activity coefficient & $\frac{d \ln f_{ \pm}}{d \ln c_{e}}=\frac{0.24 c_{e}^{0.5}-0.982[1.0-0.0052(0.966 \theta-294)] c_{e}^{1.5}}{t_{+}-1.0}$ \\
\hline
\end{tabular}

Table 4: List of parameters and properties of electrolyte for the simulation [33].

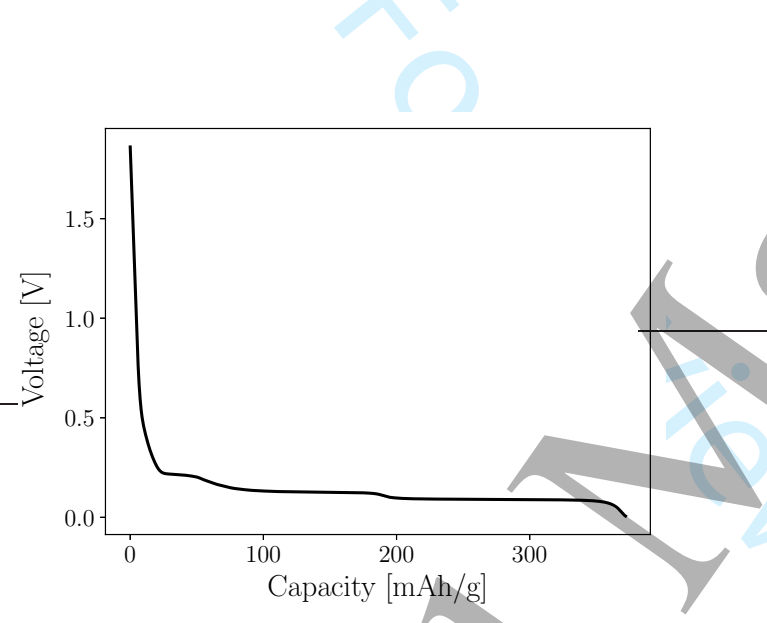

(a) graphite

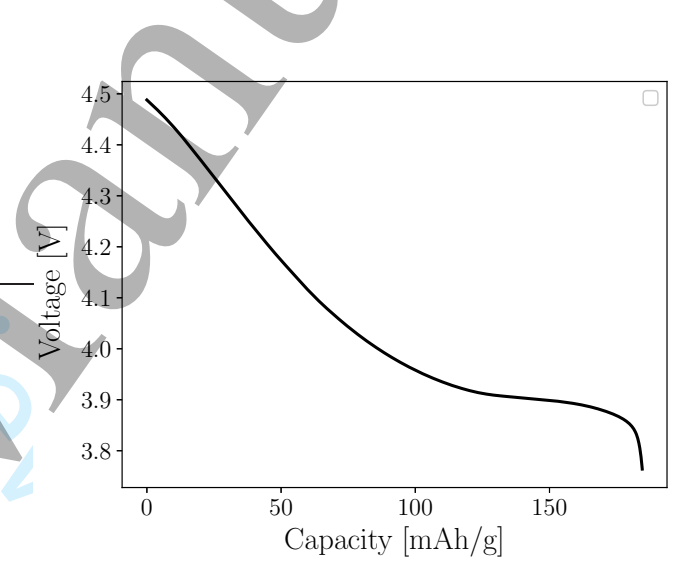

(b) $\mathrm{LCO}$

Figure 4: Measured OCP for graphite and $\mathrm{LiCoO}_{2}$ electrodes used in the $\mathrm{LG} \mathrm{G5}$ cells, which are obtained by averaging $\mathrm{C} / 50$ charge and discharge curves measured in coin cells with Li counter electrodes at $25^{\circ} \mathrm{C}$.

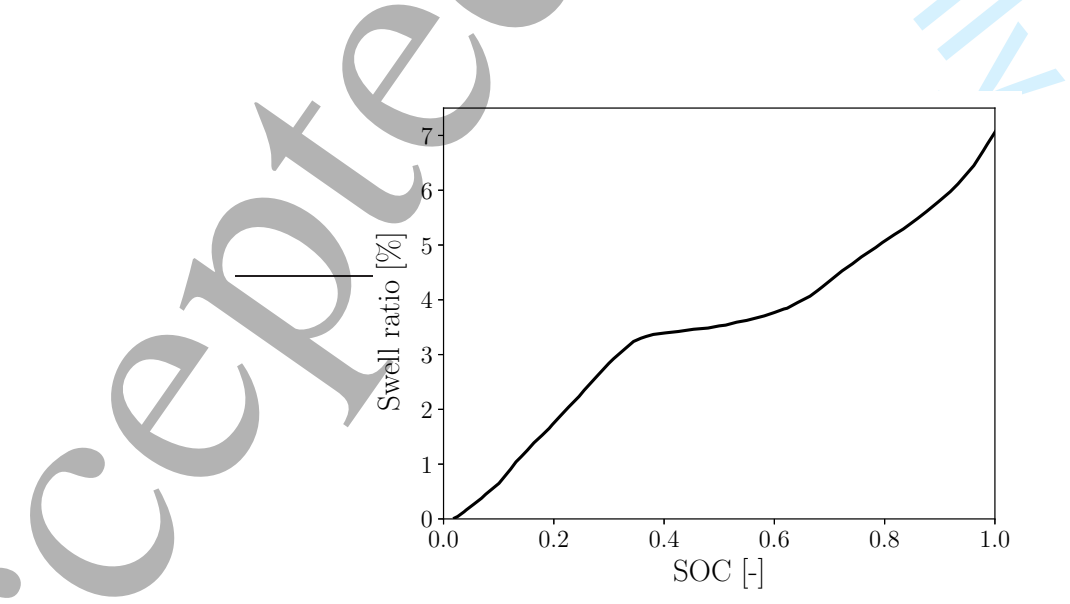

Figure 5: Measured thickness change ratio of $\mathrm{Li}_{\mathrm{x}} \mathrm{C}_{6}$ during the delithiation procedure for a pouch cell that has a graphite volume fraction of $61 \pm 1 \%$ [47]. 
A PREPRINT - JANUARY 19, 2021

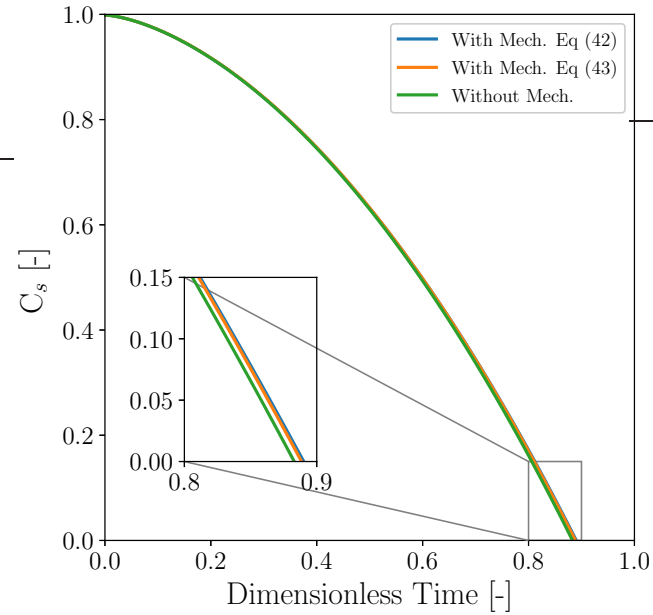

(a) surface concentration

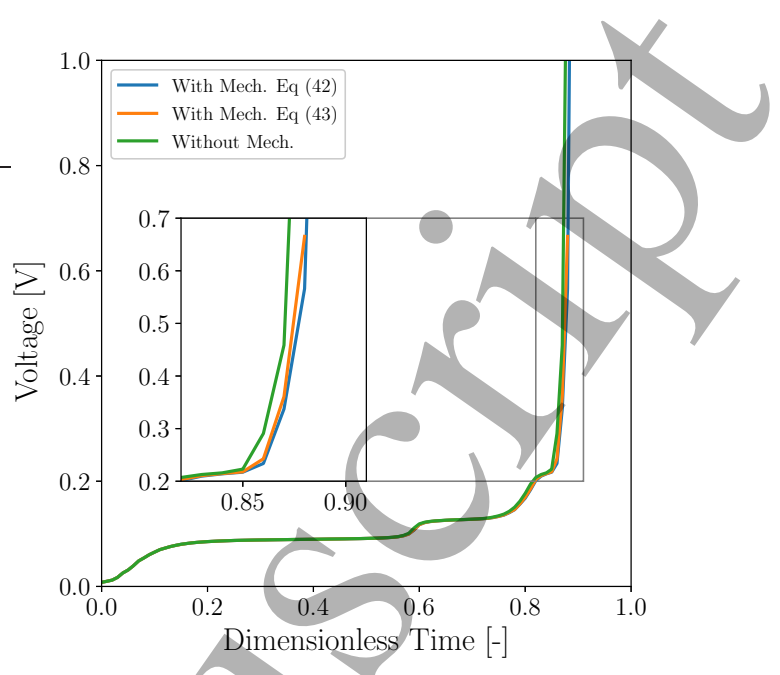

(b) surface potential

Figure 6: Comparison of surface concentration between the case neglecting mechanical effects and the case considering mechanical coupling, but using different formulations, (42) and (43). The surface potential associated with the surface concentration is shown on the right, where both plots show negligible difference among these three cases. In these simulations, the dimensionless time $\tau$ is defined as $\tau=\left(D_{s} t\right) /\left(R_{p}^{0}\right)^{2}$. Ad dimensionless pore wall flux $\delta(\tau)=\tau$ is used with $\delta(\tau)=J_{n} R_{p}^{0} / D_{s} C_{s}^{\max }[31]$.

\section{Results}

\subsection{Diffusion in active solid particles}

The mass balance equations for active particles summarized in Table 2 are obtained based on (43), which is a simplified form of (42). In (43), an averaged volume change ratio $\overline{\mathcal{J}}_{\text {active }}$ is used to solve for $\mathrm{Li}$ concentration instead of a radial location dependent volume change ratio $\mathcal{J}_{\text {active. }}$. To evaluate the consequence on the surface concentration from this assumption, (42) and (43) are solved with finite element methods (FEM) in the two dimensional setting for a spherical particle. The solid diffusion in the spherical particle without mechanical deformation is also solved with FEM for comparison. A dimensionless analysis as carried out in [31] is conducted. A surface flux linearly dependent on the dimensionless pore wall flux defined in [31] is applied to the particle surface. The surface concentration and its associated surface potential for the three cases are plotted in Fig. 6 $(a, b)$, which show negligible difference for the three equations. Such results imply that the swelling the active particles has little impact on the Li diffusion in them. Despite of this observation, Eq. (43) is used for the remaining mechanically coupled simulations.

\subsection{Discharge study of a commercial cell}

In this section, we simulate the discharge process of LG G5 cells at $\mathrm{C} / 1, \mathrm{C} / 2, \mathrm{C} / 3, \mathrm{C} / 5$, and $\mathrm{C} / 10$ with the new formulations. Considering the fact that the cell has a very small Biot number [73], the C-rates are small, and the cells were tested under forced convection, an isothermal condition is assumed for all the simulations. The simulated thickness ratio change of the cell is compared with the measured value in Fig. 7] where a reasonably good match is observed. Because the swelling relations use for graphite and $\mathrm{LiCoO}_{2}$ are not measured specifically for the tested LG G5 cells, the observed difference in Fig. 7 is expected. Comparison of cell voltage at different C-rates shows a good match between simulation results and experimental measurement, as plotted in Fig. 8 In fact, for smaller C-rates, mechanical deformation has little impact on the electrochemical response of the cell. Thus, the simulated discharge curves for these small C-rates from the DualFoil model without mechanical coupling will perform equally well. The mechanical effect on the cell's electrochemical response is more significant at higher C-rates, which is discussed in Section 4.3 .

\subsection{Porosity change in the SLTD}

The porosity change of the three porous regions is plotted in Fig. 9 which shows that intercalation induced swelling/shrinkage causes the anode porosity to increase somewhat and the cathode porosity to decrease slightly. 
Figure 7: Comparison of the cell thickness ratio between simulation results and experimental measurement at $\mathrm{C} / 2$, which shows that the model can predict the volume change of the cell with a reasonably good accuracy. Because the swelling relations use for graphite and $\mathrm{LiCoO}_{2}$ are not measured specifically for the tested LG G5 cells, the observed difference is expected.
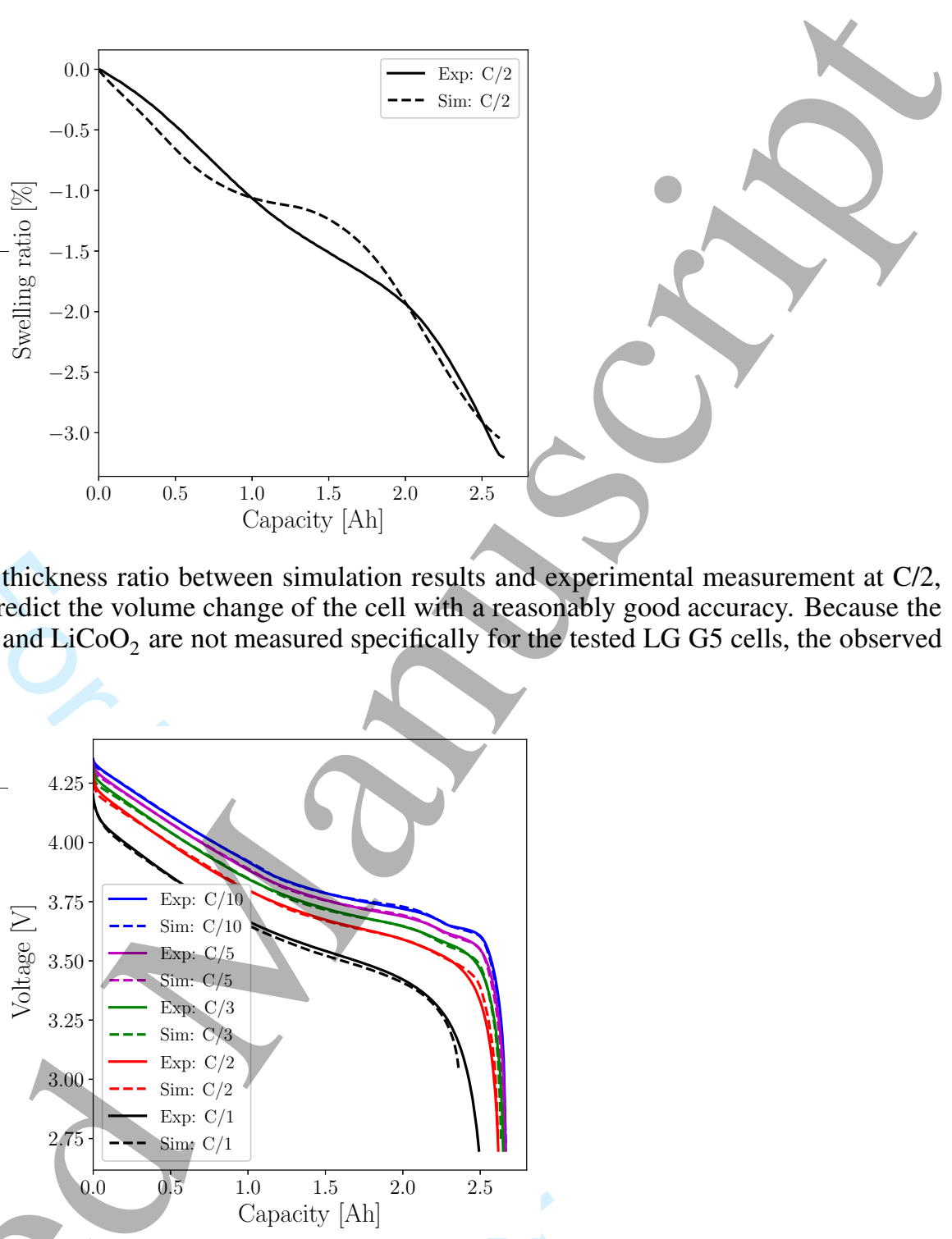

Figure 8: Comparison of voltage profile at different C-rates between simulation results and experimental measurement.

Porosity change impacts the effective transport properties of the cell via the Bruggeman relationship (1). The simulation results show that both electrodes have non-uniform porosity distribution during the simulation, which is due to the non-uniform distribution of $\mathrm{Li}$ intercalation/deintercalation rates in the electrodes. Our results show a maximum ca. $7.1 \%$ relative porosity increase in the anode region, and a maximum ca. $1.2 \%$ relative porosity decrease in the cathode region for this particular simulation. This porosity change should not be neglected in the simulation with higher Crates. For example, the discharge voltage profiles at $\mathrm{C} / 3, \mathrm{C} / 1$, and $1.2 \mathrm{C}$ from simulations with and without accounting for the mechanical effect are compared in Fig. 10. As expected, the difference is more significant at higher C-rates. This difference suggests that including mechanical effects enables more accurate simulations of the electrochemical behavior when extrapolating beyond the fitted operating parameter space to higher C-rates. Measurements of the LG G5 cells were restricted to $1 \mathrm{C}$, and hence the model parameters may not be accurate at higher C-rates. We therefore limited our simulations to $1.2 \mathrm{C}$, although this is not a general limitation of the model itself. We expand investigation of mechanical effects up to $4 \mathrm{C}$ in a subsequent work [74].

\subsection{External mechanical loading effects}

In a real cell, the jellyroll normally is wound layer by layer and is confined in the can. The constraint from the neighbor sandwich layer and the relatively rigid can will cause significant stress level change in the jellyroll. To 
A PREPRINT - JANUARY 19, 2021

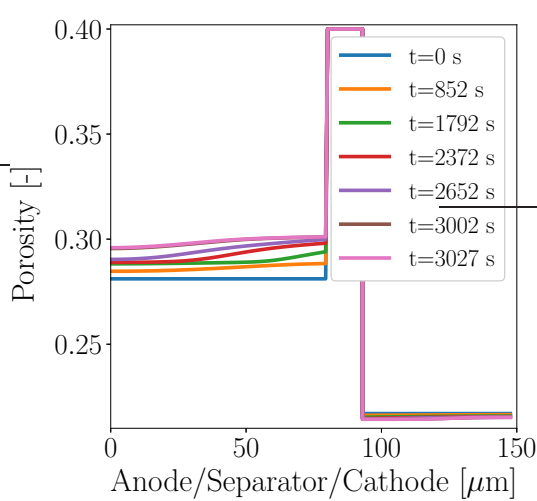

(a) all three porous regions

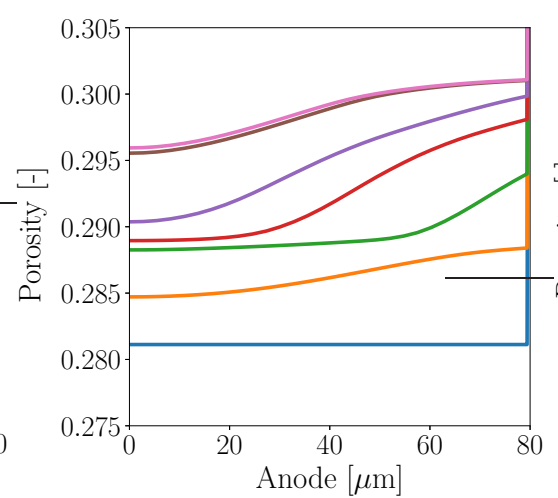

(b) anode

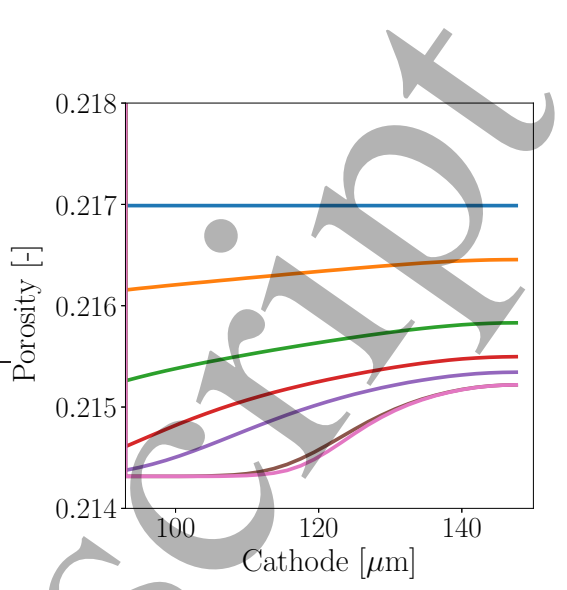

(c) cathode

Figure 9: Illustration of the porosity change of the three porous regions in a cell during a $\mathrm{C} / 1$ discharge process at different time steps without externally applied pressure. A visible non-uniform porosity distribution in both electrodes is observed, which is caused by non-uniform distribution of the Li deintercalation reaction rate.

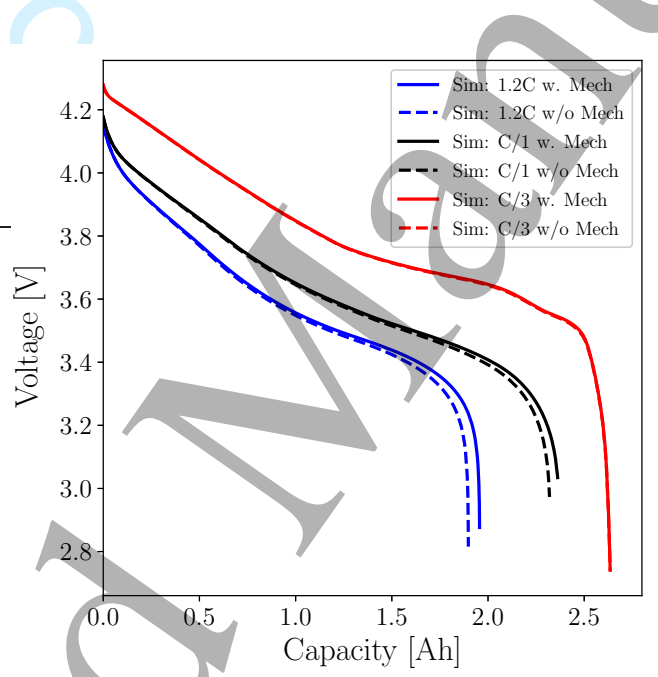

Figure 10: Comparison of voltage profile at $\mathrm{C} / 3, \mathrm{C} / 1$, and $1.2 \mathrm{C}$ from simulations with and without accounting for the mechanical effect. Difference is observed at the higher C-rate simulation.

understand how the externally applied pressure impacts cell electrochemical behavior, different normalized external pressures are applied to the cell at $\mathrm{C} / 3, \mathrm{C} / 1$, and $1.2 \mathrm{C}$ rates. The voltage profiles are shown in Fig. 11, which show that mechanical deformation has evident impact on cell electrochemical behavior at higher C-rates. This is mainly because that, at higher C-rates, Li-ion diffusion in the electrolyte is the major limiting factor, whereas porosity change can significantly impact the diffusion process via the Bruggeman relationship (1).

\section{Conclusion}

In this work, a new coupled electrochemomechanical model is presented to account for the porosity change and the transport distance change due to Li intercalation and externally applied pressure. Though carefully designed experiments are needed to thoroughly validate the proposed model, the preliminary results from a parameterized commercial LG G5 smartphone battery have shown promising performance, where the new model can accurately describe cell behavior at different discharge C-rates and reveal the potential impact of mechanical deformation on the cell's electrochemical behavior at high $\mathrm{C}$-rates. The capability to model cell behavior with varying porosity is particularly important for simulating fast charge/discharge behavior, where the Li concentration variation (equivalently, the porosity variations) along the SLTD will be much more severe than the examples studied in this work. Because of the non-negligible coupling between mechanical deformation and electrochemical behavior, we expect that in large-format cells the non- 


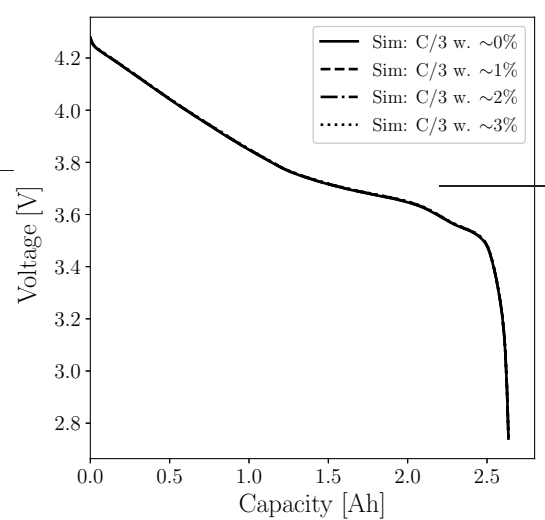

(a) $\mathrm{C} / 3$

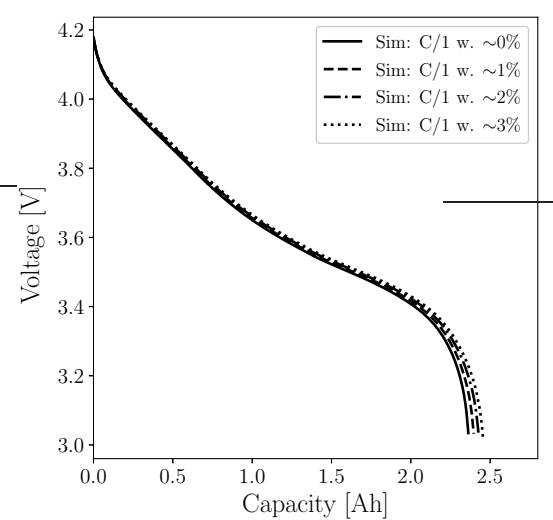

(b) $\mathrm{C} / 1$

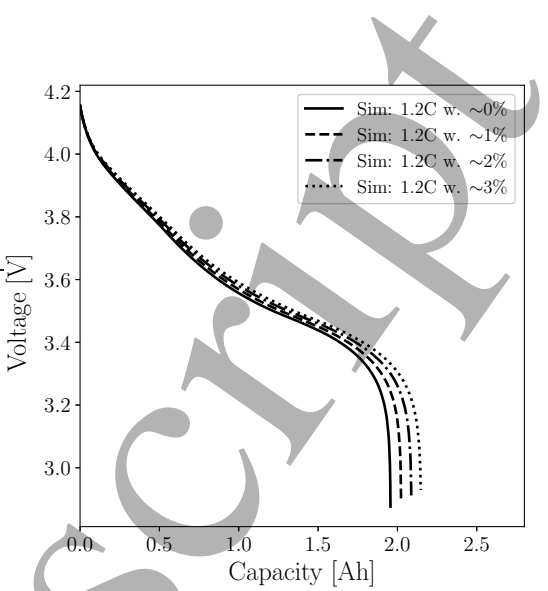

(c) $1.2 \mathrm{C}$

Figure 11: Comparison of voltage profiles for $\mathrm{C} / 3, \mathrm{C} / 1$, and $1.2 \mathrm{C}$ discharge under different externally applied pressure, with the legend $\sim 0 \%-\sim 3 \%$ indicating the equivalent level of externally applied elastic strain. The simulation results show that mechanical deformation has evident impact on cell electrochemical behavior at higher C-rates.

uniform deformation might significantly impact cell electrochemical performance and possibly the aging process. A 3D electro-chemo-thermo-mechanical model has been developed to resolve the heterogeneity inside the battery by the authors, which is published in [46].

\section{Acknowledgement}

This work was sponsored in part by Robert Bosch LLC through Bosch Energy Research Network (BERN) Grant No. 01.01.MS.17. C.L. acknowledges also support from the National Science Foundation through grant CMMI-1911836.

\section{Nomenclature}

$a_{0} \quad$ - initial active interfacial area per unit electrode volume (specific interfacial area), $1 / \mathrm{m}$

$c_{e} \quad \mathrm{Li}^{+}$concentration of electrolyte in the current configuration, $\mathrm{mol} / \mathrm{m}^{3}$

$c_{s} \quad \bullet$ surface solid-phase Li concentration, $\mathrm{mol} / \mathrm{m}^{3}$

$C_{s}^{\max } \bullet$ maximum surface concentration in the reference configuration, $\mathrm{mol} / \mathrm{m}^{3}$

$\bar{C} \bullet$ volume-averaged solid-phase $\mathrm{Li}$ concentration in the reference configuration, $\mathrm{mol} / \mathrm{m}^{3}$

$C_{e} \quad \bullet \mathrm{Li}^{+}$concentration of electrolyte in the reference configuration, $\mathrm{mol} / \mathrm{m}^{3}$

$C_{s} \quad \bullet$ Li surface concentration of active particles in the reference configuration, $\mathrm{mol} / \mathrm{m}^{3}$

$D_{s} \quad \bullet$ solid-phase diffusion coefficient, $\mathrm{m}^{2} / \mathrm{s}$

$D_{e, \text { eff }} \bullet$ effective electrolyte diffusion coefficient, $\mathrm{m}^{2} / \mathrm{s}$

$E \quad \bullet$ Young's modulus, $\mathrm{N} / \mathrm{m}^{2}$

$f_{ \pm} \quad \bullet$ salt activity coefficient

F $\quad$ Faraday's constant, $96485 \mathrm{C} / \mathrm{mol}$

$\boldsymbol{F} \bullet$ deformation gradient

$\boldsymbol{F}_{\text {active }} \bullet$ deformation gradient of active particles

$\boldsymbol{i}_{e} \quad$ electrolyte-phase current density in the current configuration, $\mathrm{A} / \mathrm{m}^{2}$

$i_{s} \quad \bullet$ solid-phase current density in the current configuration, $\mathrm{A} / \mathrm{m}^{2}$

$\boldsymbol{I}_{e} \quad$ electrolyte-phase current density in the reference configuration, $\mathrm{A} / \mathrm{m}^{2}$

$I_{s} \quad-$ solid-phase current density in the reference configuration, $\mathrm{A} / \mathrm{m}^{2}$

$\overline{\mathcal{J}}_{\text {active }} \bullet$ averaged Jacobian of active particles 
A PREPRINT - JANUARY 19, 2021

\section{References}

[1] Rong Xu and Kejie Zhao. Electrochemomechanics of Electrodes in Li-Ion Batteries: A Review. Journal of Electrochemical Energy Conversion and Storage, 13(3):030803, 2016.

[2] Ying Zhao, Peter Stein, Yang Bai, Mamun Al-Siraj, Yangyiwei Yang, and Bai Xiang Xu. A review on modeling of electro-chemo-mechanics in lithium-ion batteries. Journal of Power Sources, 413(October 2018):259-283, 2019.

[3] Jake Christensen. Modeling Diffusion-Induced Stress in Li-Ion Cells with Porous Electrodes. Journal of The Electrochemical Society, 157(3):A366, 2010.

[4] Jake Christensen and John Newman. Stress generation and fracture in lithium insertion materials. Journal of Solid State Electrochemistry, 10(5):293-319, 2006.

[5] Candace K. Chan, Hailin Peng, Gao Liu, Kevin McIlwrath, Xiao Feng Zhang, Robert A Huggins, and Yi Cui. High-performance lithium battery anodes using silicon nanowires. Nature Nanotechnology, 3(1):31-35, 2008. 
[6] B. Laforge, L. Levan-Jodin, R. Salot, and A. Billard. Study of Germanium as Electrode in Thin-Film Battery. Journal of The Electrochemical Society, 155(2):A181, 2008.

[7] Matthew T. McDowell, Ill Ryu, Seok Woo Lee, Chong-Min Wang, William D. Nix, and Yi Cui. Studying the kinetics of crystalline silicon nanoparticle lithiation with in situ transmission electron microscopy. Advanced Materials, 24(45):6034-6041, 2012.

[8] Godfrey Sikha, Branko N. Popov, and Ralph E. White. Effect of Porosity on the Capacity Fade of a Lithium-Ion Battery. Journal of The Electrochemical Society, 151(7):A1104, 2004.

[9] John Cannarella and Craig B. Arnold. Stress evolution and capacity fade in constrained lithium-ion pouch cells. Journal of Power Sources, 245:745-751, 2014.

[10] Xuning Feng, Minggao Ouyang, Xiang Liu, Languang Lu, Yong Xia, and Xiangming He. Thermal runaway mechanism of lithium ion battery for electric vehicles: A review. Energy Storage Materials, 2018.

[11] John Newman and William Tiedemann. Porous-electrode theory with battery applications. AIChE Journal, 21(1):25-41, 1975.

[12] Marc Doyle, Thomas F Fuller, and John Newman. Modeling of Galvanostatic Charge and Discharge of the Lithium/Polymer/Insertion Cell. Journal of The Electrochemical Society, 140(6):1526, 1993.

[13] Thomas F Fuller, Marc Doyle, and John Newman. Simulation and Optimization of the Dual Lithium Ion Insertion Cell. Journal of The Electrochemical Society, 141(1):1, 1994.

[14] Thomas F. Fuller, Marc Doyle, and John Newman. Relaxation Phenomena in Lithium-Ion-Insertion Cells. Journal of The Electrochemical Society, 141(4):982-990, 1994.

[15] Lin Rao and John Newman. Heat-Generation Rate and General Energy Balance for Insertion Battery Systems. Journal of The Electrochemical Society, 144:2697, 1997

[16] Karen E. Thomas and John Newman. Thermal Modeling of Porous Insertion Electrodes. Journal of The Electrochemical Society, 150(2):A176, 2003.

[17] Charles Monroe and John Newman. Dendrite Growth in Lithium/Polymer Systems. Journal of The Electrochemical Society, 150(10):A1377, 2003.

[18] Charles Monroe and John Newman. The Impact of Elastic Deformation on Deposition Kinetics at Lithium/Polymer Interfaces. Journal of The Electrochemical Society, 152(2):A396, 2005.

[19] Jake Christensen and John Newman. Effect of Anode Film Resistance on the Charge/Discharge Capacity of a Lithium-Ion Battery. Journal of The Electrochemical Society, 150(11):A1416, 2003.

[20] Jake Christensen and John Newman. A Mathematical Model for the Lithium-Ion Negative Electrode Solid Electrolyte Interphase. Journal of The Electrochemical Society, 151(11):A1977, 2004.

[21] Paul Albertus, Jake Christensen, and John Newman. Modeling Side Reactions and Nonisothermal Effects in Nickel Metal-Hydride Batteries. Journal of The Electrochemical Society, 155(1):A48, 2008.

[22] John Newman and Karen E Thomas-Alyea. Electrochemical systems. John Wiley \& Sons, 2012.

[23] Jake Christensen and John Newman. A Mathematical Model of Stress Generation and Fracture in Lithium Manganese Oxide. Journal of The Electrochemical Society, 153(6):A1019, 2006.

[24] Derek Rife, Godfrey Sikha, Sumitava De, Bharatkumar Suthar, and Venkat R. Subramanian. Efficient Reformulation of Solid Phase Diffusion in Electrochemical-Mechanical Coupled Models for Lithium-Ion Batteries: Effect of Intercalation Induced Stresses. Journal of The Electrochemical Society, 160(10):A1675-A1683, 2013.

[25] Markus Klinsmann, Daniele Rosato, Marc Kamlah, and Robert M. McMeeking. Modeling crack growth during $\mathrm{Li}$ insertion in storage particles using a fracture phase field approach. Journal of the Mechanics and Physics of Solids, 92:313-344, 2016.

[26] Markus Klinsmann, Daniele Rosato, Marc Kamlah, and Robert M. McMeeking. Modeling Crack Growth during Li Extraction in Storage Particles Using a Fracture Phase Field Approach. Journal of The Electrochemical Society, 163(2):A102-A118, 2016.

[27] Yiling Dai, Long Cai, and Ralph E. White. Simulation and analysis of stress in a Li-ion battery with a blended LiMn2O4and LiNi0.8Co0.15A10.05O2cathode. Journal of Power Sources, 247:365-376, 2014.

[28] Xiaoxuan Zhang, Seok Woo Lee, Hyun-Wook Lee, Yi Cui, and Christian Linder. A reaction-controlled diffusion model for the lithiation of silicon in lithium-ion batteries. Extreme Mechanics Letters, 4:61-75, 2015.

[29] Xiaoxuan Zhang, Andreas Krischok, and Christian Linder. A variational framework to model diffusion induced large plastic deformation and phase field fracture during initial two-phase lithiation of silicon electrodes. Computer Methods in Applied Mechanics and Engineering, 312:51-77, 2016. 
[30] Zhenlin Wang, Jason B Siegel, and Krishna Garikipati. Intercalation driven porosity effects in coupled continuum models for the electrical, chemical, thermal and mechanical response of battery electrode materials. Journal of the Electrochemical Society, 164(9):A2199-A2212, 2017.

[31] Venkat R. Subramanian, Vinten D. Diwakar, and Deepak Tapriyal. Efficient Macro-Micro Scale Coupled Modeling of Batteries. Journal of The Electrochemical Society, 152(10):A2002, 2005.

[32] P. K. Leung, C. Moreno, I. Masters, S. Hazra, B. Conde, M. R. Mohamed, R. J. Dashwood, and R. Bhagat. Realtime displacement and strain mappings of lithium-ion batteries using three-dimensional digital image correlation. Journal of Power Sources, 271:82-86, 2014.

[33] Jake Christensen, David Cook, and Paul Albertus. An Efficient Parallelizable 3D Thermoelectrochemical Model of a Li-Ion Cell. Journal of the Electrochemical Society, 160(11):A2258-A2267, 2013.

[34] Von D A G Bruggeman. Berechnung verschiedener physikalischer Konstanten von heterogenen Substanzen. I. Dielektrizitätskonstanten und Leitfähigkeiten der Mischkörper aus isotropen Substanzen. Annalen der physik, 416(7):636-664, 1935.

[35] Jae Hyun Lee, Hyang Mok Lee, and Soonho Ahn. Battery dimensional changes occurring during charge/discharge cycles - Thin rectangular lithium ion and polymer cells. Journal of Power Sources, 119121:833-837, 2003.

[36] J B Nelson and D P Riley. The thermal expansion of graphite from 15c. to 800c.: Part I. Experimental. Proceedings of the Physical Society, 57(6):477-486, 1945.

[37] D. K L Tsang, B. J. Marsden, S. L. Fok, and G. Hall. Graphite thermal expansion relationship for different temperature ranges. Carbon, 43(14):2902-2906, 2005.

[38] Eric Jianfeng Cheng, Nathan John Taylor, Jeff Wolfenstine, and Jeff Sakamoto. Elastic properties of lithium cobalt oxide (LiCoO 2 ). Journal of Asian Ceramic Societies, 5(2):113-117, 2017.

[39] Tsutomu Ohzuku, Yasunobu Iwakoshi, and Keijiro Sawai. Formation of Lithium-Graphite Intercalation Compounds in Nonaqueous Electrolytes and Their Application as a Negative Electrode for a Lithium Ion (Shuttlecock) Cell. Journal of The Electrochemical Society, 140(9):2490, 1993.

[40] Jan N Reimers and J. R. Dahn. Electrochemical and in situ X-ray diffraction studies of lithium intercalation in LixCoO2. Journal of The Electrochemical Society, 139(8):2091-2097, 1992.

[41] K. R. Kganyago and P. E. Ngoepe. Structural and electronic properties of lithium intercalated graphite LiC6. Physical Review B - Condensed Matter and Materials Physics, 68(20):1-16, 2003.

[42] Veruska Malavé, J. R. Berger, Huayang Zhu, and Robert J. Kee. A computational model of the mechanical behavior within reconstructed LixCoO2Li-ion battery cathode particles. Electrochimica Acta, 130:707-717, 2014.

[43] Norio Takami, Asako Satoh, Michikazu Hara, and Takahisa Ohsaki. Structural and kinetic characterization of lithium intercalation into carbon anodes for secondary lithium batteries. Journal of The Electrochemical Society, 142(2):371-379, 1995.

[44] Nalin A. Chaturvedi, Reinhardt Klein, Jake Christensen, Jasim Ahmed, and Aleksandar Kojic. Algorithms for Advanced Battery-Management Systems: Modeling, estimation, and control challenges for lithium-ion batteries. IEEE Control Systems, 30(3):49-68, 2010.

[45] Nalin A Chaturyedi, Jake Christensen, Reinhardt Klein, and Aleksandar Kojic. Approximations for Partial Differential Equations Appearing in Li-Ion Battery Models. In Dynamic Systems and Control Conference, volume 56123, page V001T05A004, 2013.

[46] Xiaoxuan Zhang, Sergei Chumakov, Xiaobai Li, Markus Klinsmann, Sun Ung Kim, Christian Linder, and Jake Christensen. An electro-chemo-thermo-mechanical coupled three-dimensional computational framework for Liion batteries. Journal of The Electrochemical Society, 167(16):160542, 2020.

[47] B. Rieger, S. Schlueter, S. V. Erhard, J. Schmalz, G. Reinhart, and A. Jossen. Multi-scale investigation of thickness changes in a commercial pouch type lithium-ion battery. Journal of Energy Storage, 6:213-221, 2016.

[48] Tyler DuBeshter, Puneet K. Sinha, Alex Sakars, Gerald W. Fly, and Jacob Jorne. Measurement of Tortuosity and Porosity of Porous Battery Electrodes. Journal of The Electrochemical Society, 161(4):A599-A605, 2014.

[49] Siqi Tang, Zhixing Wang, Huajun Guo, Jiexi Wang, Xinhai Li, and Guochun Yan. Simulating the distribution of electrochemical characteristics: A case study of $4.35 \mathrm{~V} \mathrm{LiCoO} /$ graphite pouch cell. Electrochimica Acta, 354:136634, 2020. 
[50] M. D. Levi and D. Aurbach. Diffusion coefficients of lithium ions during intercalation into graphite derived from the simultaneous measurements and modeling of electrochemical impedance and potentiostatic intermittent titration characteristics of thin graphite electrodes. Journal of Physical Chemistry B, 101(23):4641-4647, 1997.

[51] Kristin Persson, Vijay A. Sethuraman, Laurence J. Hardwick, Yoyo Hinuma, Ying Shirley Meng, Anton Van Der Ven, Venkat Srinivasan, Robert Kostecki, and Gerbrand Ceder. Lithium diffusion in graphitic carbon. Journal of Physical Chemistry Letters, 1(8):1176-1180, 2010.

[52] Myounggu Park, Xiangchun Zhang, Myoungdo Chung, Gregory B. Less, and Ann Marie Sastry. A review of conduction phenomena in Li-ion batteries. J. Power Sources, 195:7904-7929, 2010.

[53] Kehua Dai, Zhihui Wang, Guo Ai, Hui Zhao, Wen Yuan, Xiangyun Song, Vincent Battaglia, Chengdong Sun, Kai Wu, and Gao Liu. The transformation of graphite electrode materials in lithium-ion batteries after cycling. Journal of Power Sources, 298:349-354, 2015.

[54] Barzin Rajabloo, Ali Jokar, Martin Désilets, and Marcel Lacroix. An Inverse Method for Estimating the Electrochemical Parameters of Lithium-Ion Batteries. Journal of The Electrochemical Society, 164(2):A99-A105, 2017.

[55] Venkat R. Subramanian, Vijayasekaran Boovaragavan, Venkatasailanathan Ramadesigan, and Mounika Arabandi. Mathematical Model Reformulation for Lithium-Ion Battery Simulations: Galvanostatic Boundary Conditions. Journal of The Electrochemical Society, 156(4):A260, 2009.

[56] Martin Ebner and Vanessa Wood. Tool for Tortuosity Estimation in Lithium Ion Battery Porous Electrodes. Journal of The Electrochemical Society, 162(2):A3064-A3070, 2015.

[57] J Turner, S Allu, S Gorti, S Kalnaus, A Kumar, D Lebrun-Grandie, S Pannala, S Simunovic, S Slattery, and $\mathrm{H}$ Wang. Crash Models for Advanced Automotive Batteries. Technical report, Oak Ridge National Laboratory, 2015.

[58] Masashi Okubo, Yoshinori Tanaka, Haoshen Zhou, Tetsuichi Kudo, and Itaru Honma. Determination of activation energy for Li ion diffusion in electrodes. Journal of Physical Chemistry B, 113(9):2840-2847, 2009.

[59] R. Fallahzadeh and N. Farhadian. Molecular dynamics simulation of lithium ion diffusion in LiCoO2 cathode material. Solid State Ionics, 280:10-17, 2015.

[60] H Xia, Y S Meng, L Lu, Gerbrand Ceder, and Singapore-mit Alliance. Electrochemical behavior and Li Diffusion study of LiCoO 2 thin film electrodes prepared by PLD. Scientific Commons, page http://en.scientificcommons.org/20613936, 2010.

[61] Young Il Jang, Bernd J. Neudecker, and Nancy J. Dudney. Lithium diffusion in LixCoO2 $(0.45<\mathrm{x}<0.7)$ intercalation cathodes. Electrochemical and Solid-State Letters, 4(6):2-6, 2001.

[62] Siqi Tang, Zhixing Wang, Huajun Guo, Jiexi Wang, Xinhai Li, and Guochun Yan. Systematic parameter acquisition method for electrochemical model of 4.35 V LiCoO2 batteries. Solid State Ionics, 343:115083, 2019.

[63] Maria Angeles Cabanero, Nicola Boaretto, Manuel Roder, Jana Muller, Josef Kallo, and Arnulf Latz. Direct Determination of Diffusion Coefficients in Commercial Li-Ion Batteries. J. Electrochem. Soc., 165:A847-A855, 2018.

[64] Linsen Li, Randall M. Erb, Jiajun Wang, Jun Wang, and Yet Ming Chiang. Fabrication of Low-Tortuosity Ultrahigh-Area-Capacity Battery Electrodes through Magnetic Alignment of Emulsion-Based Slurries. Adv. Energy Mater., 9:1-7, 2019.

[65] Marc Doyle and Yuris Fuentes. Computer Simulations of a Lithium-Ion Polymer Battery and Implications for Higher Capacity Next-Generation Battery Designs. J. Electrochem. Soc., 150:A706, 2003.

[66] Bernhard Rieger, Simon V. Erhard, Katharina Rumpf, and Andreas Jossen. A New Method to Model the Thickness Change of a Commercial Pouch Cell during Discharge. Journal of The Electrochemical Society, 163(8):A1566-A1575, 2016.

[67] Fernando Henrique Pavoni, Lucas Evangelista Sita, Caroline Santana dos Santos, Stephany Pires da Silva, Paulo Rogério Catarini da Silva, and Jair Scarminio. LiCoO2 particle size distribution as a function of the state of health of discarded cell phone batteries. Powder Technology, 326:78-83, 2018.

[68] Indrajeet V. Thorat, David E. Stephenson, Nathan A. Zacharias, Karim Zaghib, John N. Harb, and Dean R. Wheeler. Quantifying tortuosity in porous Li-ion battery materials. Journal of Power Sources, 188(2):592-600, 2009.

[69] Donal P. Finegan, Samuel J. Cooper, Bernhard Tjaden, Oluwadamilola O. Taiwo, Jeff Gelb, Gareth Hinds, Dan J.L. Brett, and Paul R. Shearing. Characterising the structural properties of polymer separators for lithiumion batteries in 3D using phase contrast X-ray microscopy. Journal of Power Sources, 333:184-192, 2016. 
[70] Corey T. Love. Thermomechanical analysis and durability of commercial micro-porous polymer Li-ion battery separators. Journal of Power Sources, 196(5):2905-2912, 2011.

[71] LG G5 Battery - OEM Batteries For Sale I LG USA. \url\{http://www.lg.com/us/mobile-accessories/lg-BL42D1F-g5-battery\}.

[72] Ki Yong Oh, Jason B. Siegel, Lynn Secondo, Sun Ung Kim, Nassim A. Samad, Jiawei Qin, Dyche Anderson, Krishna Garikipati, Aaron Knobloch, Bogdan I. Epureanu, Charles W. Monroe, and Anna Stefanopoulou. Rate dependence of swelling in lithium-ion cells. Journal of Power Sources, 267:197-202, 2014.

[73] Xiaoxuan Zhang, Reinhardt Klein, A Subbaraman, S Chumakov, X Li, Jake Christensen, Christian Linder, and S U Kim. Evaluation of convective heat transfer coefficient and specific heat capacity of a lithium-ion battery using infrared camera and lumped capacitance method. Journal of Power Sources, 412:552-558, 2019.

[74] Yitao Qiu, Xiaoxuan Zhang, Camille Usubelli, Christian Linder, and Jake Christensen. Analysis of thermal and mechanical effects on Lithium plating in automotive cells with large energy capacity. to be submitted, 2021. 